\title{
A Systematic Review of Xuezhikang, an Extract from Red Yeast Rice, for Coronary Heart Disease Complicated by Dyslipidemia
}

\author{
Qinghua Shang, ${ }^{1}$ Zhaolan Liu, ${ }^{2}$ Keji Chen, ${ }^{3}$ Hao Xu, ${ }^{3}$ and Jianping Liu ${ }^{2}$ \\ ${ }^{1}$ Graduate School of Chinese Medicine, Beijing University, Beijing 100029, China \\ ${ }^{2}$ Centre for Evidence-Based Chinese Medicine, Beijing University of Chinese Medicine, Beijing 100029, China \\ ${ }^{3}$ Cardiovascular Disease Center, Xiyuan Hospital, China Academy of Chinese Medical Sciences, Beijing 100091, China
}

Correspondence should be addressed to Hao Xu, xuhaotcm@gmail.com and Jianping Liu, jianping_l@hotmail.com

Received 14 November 2011; Accepted 9 January 2012

Academic Editor: Myeong Soo Lee

Copyright (C) 2012 Qinghua Shang et al. This is an open access article distributed under the Creative Commons Attribution License, which permits unrestricted use, distribution, and reproduction in any medium, provided the original work is properly cited.

Objective. This systematic review aims to evaluate the benefit and side effect of Xuezhikang for coronary heart disease (CHD) complicated by dyslipidemia. Methods. All randomized clinical trials (RCTs) with Xuezhikang as a treatment for CHD combined with dyslipidemia were considered for inclusion. Data extraction and analyses and quality assessment were conducted according to the Cochrane standards. Results. We included 22 randomized trials. Xuezhikang showed significant benefit on the incidence of all-cause deaths, CHD deaths, myocardial infarction, and revascularization as compared with placebo based on conventional treatment for CHD. It remarkably lowered total cholesterol (TC), triglyceride (TG), and low-density lipoprotein-cholesterol (LDLC) as compared with the placebo or inositol nicotinate group, which was similar to statins group. Xuezhikang also raised highdensity lipoprotein cholesterol (HDL-C) compared to placebo or no intervention, which was similar to Inositol nicotinate and slightly inferior to statins. The incidence of adverse events did not differ between the Xuezhikang and control group. Conclusions. Xuezhikang showed a comprehensive lipid-regulating effect and was safe and effective in reducing cardiovascular events in CHD patients complicated by dyslipidemia. However, more rigorous trials with high quality are needed to give high level of evidence.

\section{Introduction}

Coronary heart disease (CHD) is one of the most serious diseases with high incidence and mortality. Dyslipidemia contributes greatly to the formation and progression of atherosclerosis (AS), which plays a dominant role in leading to CHD. Patients with CHD are also commonly complicated with dyslipidemia. Modulating dyslipidemia actively, especially lowering low-density lipoprotein-cholesterol (LDL-C) by statins, has been demonstrated to be very crucial to prevent AS and reduce the morbidity and mortality of CHD. Most recently, the updated ESC/EAS guidelines for management of dyslipidemia [1] further highlighted the aggressive lipid-lowering strategy in subjects with documented coronary vascular disease (CVD) or previous myocardial infarction (MI). However, the application of statins might be restricted by the adverse effect on the liver function and creatine kinase, especially in patients with old age, multiple comorbid diseases, high-dose statins, or a combination lipidlowering therapy. Thus it is of great clinical significance to find an effective but safer alternative therapy in CHD patients complicated by dyslipidemia.

Xuezhikang is a partially purified extract of fermented red yeast rice (Monascus purpureus). It is composed of 13 kinds of natural statins, unsaturated fatty acids, ergosterol, amino acids, flavonoids, alkaloid, trace element, and so forth. The health enhancing qualities of this yeast have been introduced and used in China for over two thousand years. At latest systematic review indicated the beneficial effects of Xuezhikang in the treatment of hyperlipidemia [2]. Therefore, Xuezhikang has been recommended in a guideline for China adult dyslipidemia prevention [3]. Recently, clinical benefits of Xuezhikang were also found in CHD patients combined with dyslipidemia in some randomized controlled trials [4-6]. This systematic review aims to evaluate the benefit and side effect of Xuezhikang, a potential 
TABLE 1: Definition of dyslipidemia or treatment goal of patients with CHD or equivalents on serum lipid level.

\begin{tabular}{|c|c|}
\hline Orgination & Definition of dyslipidemia or treatment goal of Patients with CHD or equivalents on serum lipid level \\
\hline ATP I 1988 [14] & $\begin{array}{l}\text { Ideal lipid level: TC }<5.17 \mathrm{mmol} / \mathrm{L}(200 \mathrm{mg} / \mathrm{dL}) ; \mathrm{LDL}-\mathrm{C}<3.36 \mathrm{mmol} / \mathrm{L}(130 \mathrm{mg} / \mathrm{dL}) . \text { Patients with } \\
\mathrm{HDL}-\mathrm{C}<0.9 \mathrm{mmol} / \mathrm{L}(35 \mathrm{mg} / \mathrm{dL}) \text { were defined unmoral. The definition of dyslipidemia was } \\
\text { according to the level of LDL-C }\end{array}$ \\
\hline ATP II $1993[15]$ & Treatment goal: LDL-C $\leqq 2.6 \mathrm{mmol} / \mathrm{L}(100 \mathrm{mg} / \mathrm{dL})$ \\
\hline $\begin{array}{l}\text { Ministry of Health of the People's } \\
\text { Republic of China } 1993 \text { [8] }\end{array}$ & The treatment goal was not introduced \\
\hline CADPS $1997[16]$ & $\begin{array}{l}\text { Treatment goal: TC }<4.68 \mathrm{mmol} / \mathrm{L}(180 \mathrm{mg} / \mathrm{dL}) ; \mathrm{TG}<1.7 \mathrm{mmol} / \mathrm{L}(150 \mathrm{mg} / \mathrm{dL}) \\
\mathrm{LDL}-\mathrm{C}<2.6 \mathrm{mmol} / \mathrm{L}(100 \mathrm{mmol} / \mathrm{L})\end{array}$ \\
\hline ATP III 2001 [17] & Treatment goal: LDL-C $<2.6 \mathrm{mmol} / \mathrm{L}(100 \mathrm{mg} / \mathrm{dL})$ \\
\hline Implication of ATP III 2004 [18] & $\begin{array}{l}\text { Treatment goal: LDL-C }<2.6 \mathrm{mmol} / \mathrm{L}(100 \mathrm{mg} / \mathrm{dL}) ; \text { the optional goal: } \mathrm{LDL}-\mathrm{C}<1.8 \mathrm{mmol} / \mathrm{L} \\
(70 \mathrm{mg} / \mathrm{dL})\end{array}$ \\
\hline AHA/ACC Guideline 2006 [19] & $\begin{array}{l}\text { Treatment goal: } \mathrm{LDL}-\mathrm{C}<2.6 \mathrm{mmol} / \mathrm{L}(100 \mathrm{mmol} / \mathrm{L}) \text {, and it is seasonal for lower than } 1.8 \mathrm{mmol} / \mathrm{L} \\
(70 \mathrm{mg} / \mathrm{dL})\end{array}$ \\
\hline CADPG $2007[3]$ & $\begin{array}{l}\text { Treatment goal: TC }<4.14 \mathrm{mmol} / \mathrm{L}(160 \mathrm{mg} / \mathrm{dL}) \text { and LDL-C }<2.59 \mathrm{mmol} / \mathrm{L}(100 \mathrm{mg} / \mathrm{dL}) \text { for CHD or } \\
\text { equivalents } \\
\text { Treatment goal: TC }<3.11 \mathrm{mmol} / \mathrm{L}(120 \mathrm{mg} / \mathrm{dL}) ; \mathrm{LDL}-\mathrm{C}<2.07 \mathrm{mmol} / \mathrm{L}(80 \mathrm{mg} / \mathrm{dL}) \text { for ACS or } \\
\text { ischemic cardiovascular disease complicated with diabetes mellitus } \\
\text { Suitable scope of HDL-C: } \geqslant 1.04 \mathrm{mmol} / \mathrm{L}(40 \mathrm{mg} / \mathrm{dL}) \text {; suitable scope of TG: }<1.7 \mathrm{mmol} / \mathrm{L} \\
(150 \mathrm{mg} / \mathrm{dL})\end{array}$ \\
\hline ESC/EAS $2011[1]$ & $\begin{array}{l}\text { In patients at very high CV risk (established CVD, type } 2 \text { diabetes, type I diabetes with target organ } \\
\text { damage, moderate to severe CKD or a score level } \geqq 10 \%) \text {, the LDL-C goal is }<1.8 \mathrm{mmol} / \mathrm{L} \\
(70 \mathrm{mg} / \mathrm{dL} \text { ) and/or } \geqslant 50 \% \mathrm{LDL}-\mathrm{C} \text { reduction when target level cannot be reached (I A } \\
\text { recommendation) }\end{array}$ \\
\hline
\end{tabular}

alternative drug of statins, for CHD patients complicated by dyslipidemia, and thus provide further evidence for clinical application.

\section{Methods}

2.1. Inclusion Criteria. Randomized controlled trials (RCTs) comparing Xuezhikang with placebo, no intervention, or established lipid-lowing agents in English or Chinese were considered. Quasirandomized trials were excluded, and the duration of the intervention was no less than four weeks. Participants of all age with CHD complicated by dyslipidemia meeting with at least one of the current or past definitions or guidelines of CHD [including acute coronary syndrome (ACS)] [7-13] and dyslipidemia (treatment goal as the lower limit, see Table 1) [14-20] were considered. Those who did not introduce diagnostic criteria in the text but stated patients with definite CHD or dyslipidemia were also included. Secondary dyslipidemia, high serum lipid level after meal, serious heart failure, and serious hepatic or renal failure were excluded.

Outcome measures include primary outcomes (including all-cause mortality, CHD mortality, incidence of MI, revascularization, and rehospitalization for unstable angina) and secondary outcomes [including serum total cholesterol (TC), triglyceride (TG), LDL-C, and-high density lipoprotein cholesterol (HDL-C)].

2.2. Search Strategy. Two reviewers searched the following databases up to September 2011 independently for the identifications of trials (publication or nonpublication): The Cochrane Library, Pubmed, Chinese Biomedical Database (CBM), China National Knowledge Infrastructure (CNKI), Chinese VIP Information (VIP), and Wanfang Databases. We used the terms as follows: coronary heart disease, CHD, coronary artery disease, angina pectoris, myocardial infarction, acute coronary syndrome, cardi*, and Xuezhikang, red yeast rice, monascus. Because of different characteristics of various databases, MeSH terms and free text terms were used regardless of the report types in full text, title, keyword, subject terms, or abstract.

2.3. Data Extraction and Quality Assessment. Two reviewers (Shang QH, Liu ZL) independently extracted data according to a data extraction form made by the authors. Disagreements were resolved by consensus or consultation from a third reviewer (Liu JP). The methodological quality of trials was assessed independently using criteria from the Cochrane Handbook for Systematic Review of Interventions, Version 5.0.1 (Shang QH, Liu ZL) [20]. We contacted with the authors if there was any doubt in randomization and blinding method. The items included random sequence generation (selection bias), allocation concealment (selection bias), blinding of participants and personnel (performance bias), blinding of outcome assessment (detection bias), incomplete outcome data (attrition bias), selective reporting (reporting bias), and other bias. We judged each item from three levels ("Yes" for a low of bias, "No" for a high risk of bias, "Unclear" otherwise), and then we assessed the trials and categorized them into three levels: low risk of bias (all the items were in low risk of bias), high risk of bias (at least 


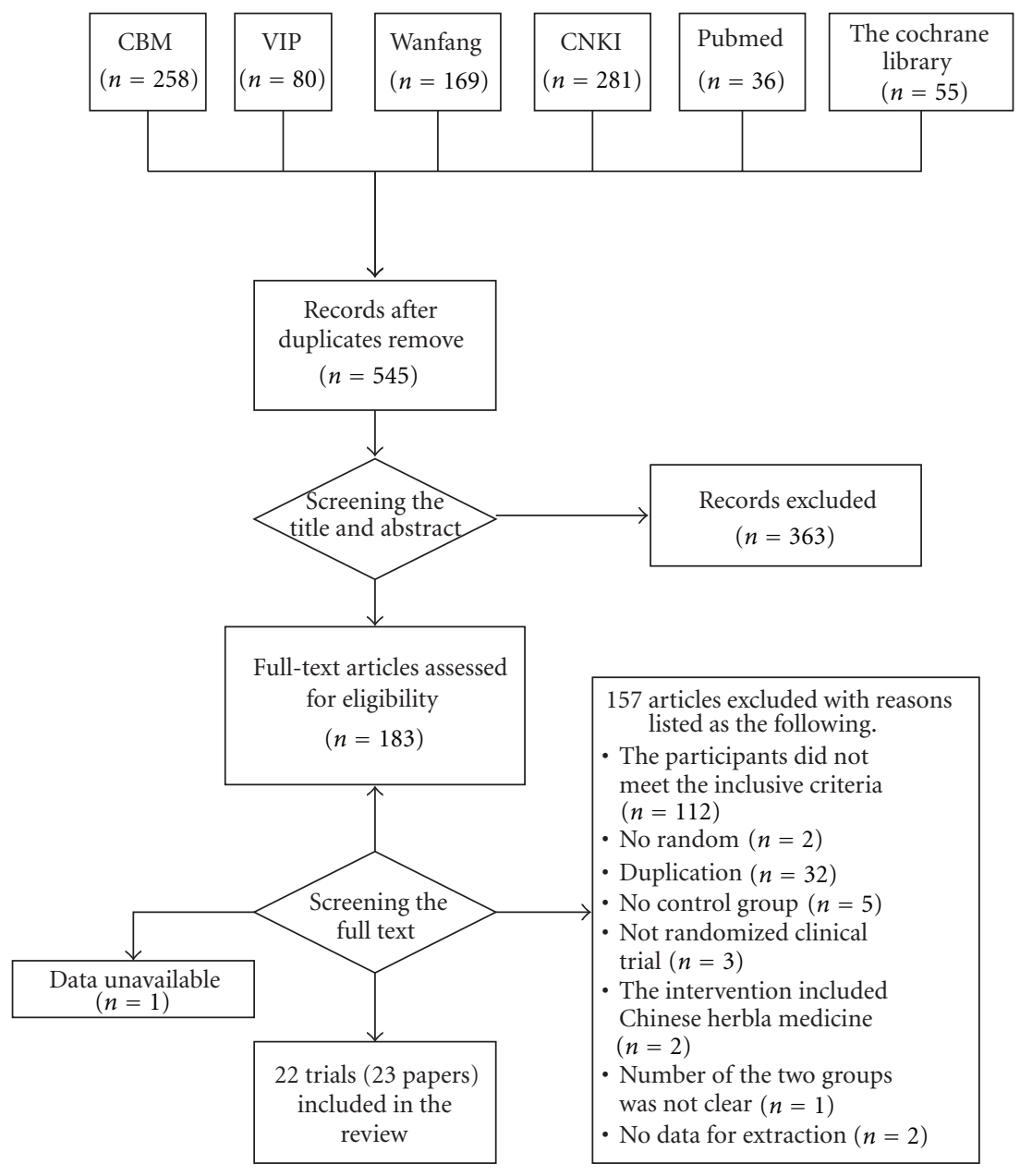

FIGURE 1: Flow chart of study selection.

one item was in high risk of bias), unclear risk of bias (at least one item was in unclear).

2.4. Data Synthesis. We used Revman 5.1 software provided by the Cochrane Collaboration for data analyses. Studies were stratified by the types of comparisons. We will express dichotomous data as risk ratio (RR) and its 95\% confidence intervals (CI). Continuous outcome will be presented as mean difference (MD) and its 95\% CI. Heterogeneity was recognized significant when $I^{2} \geq 50 \%$. Fixed effects model was used if there is no significant heterogeneity of the data; random effects model was used if significant heterogeneity existed $\left(50 \%<I^{2}<85 \%\right)$. Publication bias was explored using a funnel plot.

\section{Results}

3.1. Description of Included Trials. 22 RCTs (23 papers) [4-6, 21-39] were included, 21 papers were published in Chinese, one paper published in English, and one was unpublished as a postgraduate dissertation. The whole process of trials selection was demonstrated in Figure 1. The characteristics of included trials were listed in Table 2.

6520 Participants were included (3264 in intervention group and 3256 in control group). Two of the trials did not report the gender, and 4905 male and 1538 female were included in the other 20 trials. A total of 7 criteria of CHD (including ACS) were selected, but 6 trials did not introduce criteria of CHD but mentioned "patients with CHD were eligible to include." 3 criteria of dyslipidemia were used for 11 trials, and the other 11 trials only reported the serum lipid levels, which were categorized to dyslipidemia according to the previous and current definitions and guidelines Table 1. One trial [4] included patients with MI; five of the trials $[5,27,28,34,39]$ included patients with unstable angina; two of the trials $[6,38]$ included patients with ACS; three of the trials $[21,22,31]$ included patients with stable angina. The other 11 trials [23-26, 29, 30, 32, 33, 35-37] did not introduce the types of CHD or all types were included.

Patients in 19 trials prescribed Xuezhikang $600 \mathrm{mg}$ QD (regulation was conducted for adverse events), one trial used Xuezhikang $600 \mathrm{mg}$ TID if the serum TC or TG still higher 
after having been prescribed for 6 weeks (600 mg BID in previous 6 weeks) [30], one trial [37] prescribed Xuezhikang $300 \mathrm{mg}$ TID, and one trial [31] prescribed Xuezhikang $1200 \mathrm{mg}$ QN. The duration of treatment ranged from 4 weeks to 7 years.

There were five comparisons in the review according to various control groups. (1) Xuezhikang and conventional therapy versus conventional therapy (8 trials) $[5,6,24,29$, 33, 34, 38, 39]; (2) Xuezhikang and conventional therapy versus placebo and conventional therapy (2 trials) [4, 35]; (3) Xuezhikang and conventional therapy versus statin and conventional therapy (9 trials) [21-23, 25, 26, 28, 31, 37, 39]; (4) Xuezhikang and statin and conventional therapy versus statin and conventional therapy (2 trials) [27, 36]; (5) Xuezhikang and aspirin versus inositol nicotinate and aspirin (1 trials) [32]. One trial [39] was designed as three groups with two comparisons and Xuezhikang and conventional therapy versus conventional therapy; Xuezhikang and conventional therapy versus atorvastatin and conventional therapy.

3.2. Methodological Quality of Included Trials. According to the criteria introduced above, no trial was evaluated as having a low risk of bias. Only one trial of the 22 trials reported the method to generate the allocation sequence (random number table) in the paper [6]. After we contacted with the authors, six trials announced a correct method for allocation sequence $[4-6,31,33,35]$. One trial was assessed as having adequate concealment [35]. Two trials applied doubleblinding $[4,35]$, and two trials used single-blinding but did give us objective to be blinded $[25,37]$. One trial blinded the outcome assessors [4]. One trial reported prior sample size estimation and mentioned intention-to-treat analysis [4]. Five trials reported information on withdrawal/dropout [4, $6,22,29,32] .18$ trials $[4-6,22-27,29,31-33,35-39]$ provided baseline data for the comparability among groups. The results of the assessment of risk of bias are presented in a "risk of bias summary" figure produced by Revman 5.1 automatically Figure 2.

\subsection{Effect Estimates of Outcomes (Tables 3 and 4)}

3.3.1. All-Cause Mortality. There was only 1 trial [4] reported the all-cause mortality in the comparisons of Xuezhikang and conventional therapy versus placebo and conventional therapy [RR $0.67 ; 95 \%$ CI 0.54 to $0.83 ; 1$ trial, $n=4870$ ].

3.3.2. Mortality of CHD. There were 5 studies [4, 22, 27, $28,32]$ that presented the effect of Xuezhikang in reducing the mortality of CHD. Compared to placebo on the basis of conventional therapy, Xuezhikang showed a reduction of mortality of CHD (RR $0.69 ; 95 \%$ CI 0.54 to $0.89 ; 1$ trial, $n=4870$ ) [4]. Compared to simvastatin on the basis of conventional therapy, Xuezhikang showed no significant difference in mortality of CHD (RR 0.26 ; 95\% CI 0.06 to $1.21 ; 2$ trial, $n=220)[22,28]$. Compared to no treatment on the basis of simvastatin and conventional therapy, Xuezhikang showed no effect in reducing mortality of CHD (RR $0.33 ; 95 \%$ CI 0.01 to $7.80 ; 1$ trial, $n=48$ ) [27].

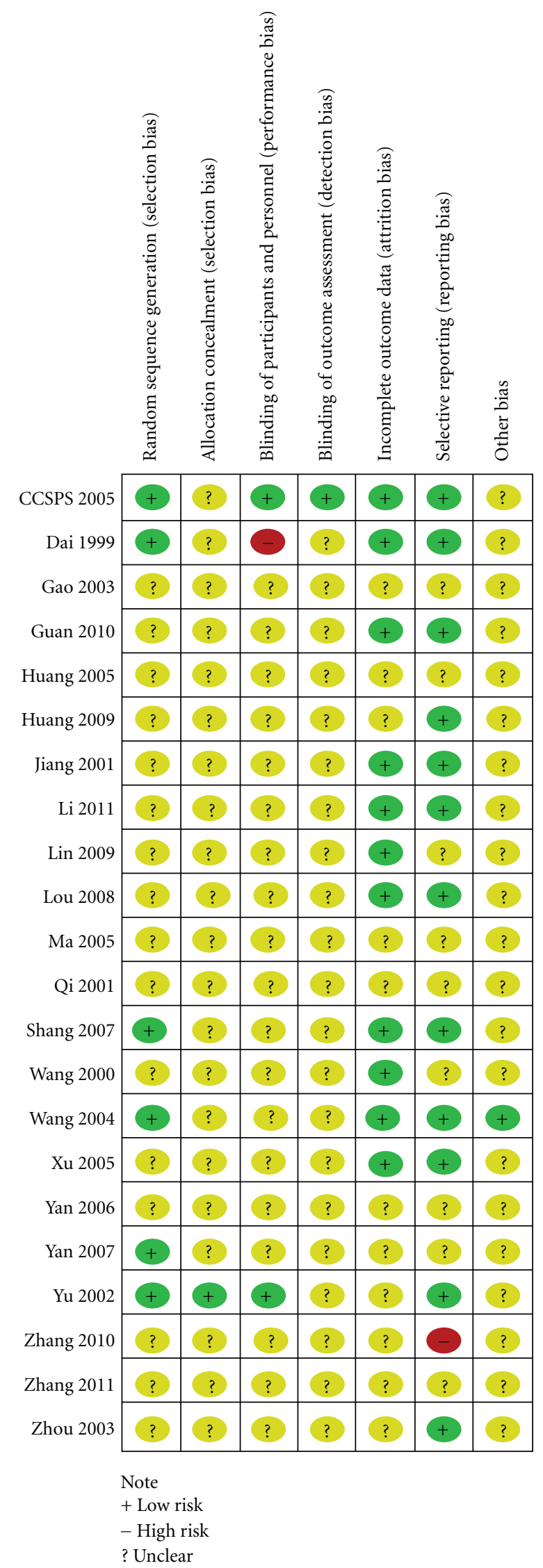

Figure 2: Risk of bias summary. 
Compared with inositol nicotinate on the basis of aspirin, Xuezhikang showed no significant difference in mortality of CHD (RR 0.15; 95\% CI 0.02 to $1.18 ; 1$ trial, $n=122$ ) [32].

3.3.3. Incidence of MI. There were 3 studies reporting CHD events in 3 different comparisons. Compared with placebo on the basis of conventional therapy, Xuezhikang showed a reduction of morbidity of MI (RR 0.39 ; 95\% CI 0.28 to 0.55 ; 1 trial, $n=4870$ ) [4]. Compared with simvastatin on the basis of conventional therapy, Xuezhikang showed no significant difference (RR $0.95 ; 95 \%$ CI 0.30 to $3.05 ; 1$ trial, $n=$ 84) [28]. In comparisons of Xuezhikang and simvastatin and conventional therapy versus simvastatin and conventional therapy, Xuezhikang showed no effect in reducing incidence of MI (RR 0.20; 95\% CI 0.01 to 3.96; 1 trial, $n=48$ ) [27].

3.3.4. Revascularization. Revascularization included percutaneous coronary intervention (PCI) and coronary artery bypass graft (CABG). There were 2 studies $[4,28]$ reporting revascularization in 2 different comparisons. Compared with placebo on the basis of conventional therapy, Xuezhikang showed a significant reduction of revascularization (RR 0.67; 95\% CI 0.50 to $0.89 ; 1$ trial, $n=4870$ ) [4]. Compared with simvastatin on the basis of conventional therapy, Xuezhikang showed no significant difference (RR 1.14; 95\% CI 0.38 to 3.46; 1 trial, $n=84$ ) [28].

3.3.5. Rehospitalization for Unstable Angina. There were 2 trials $[27,28]$ reporting rehospitalization in 2 different comparisons. Compared with simvastatin on the basis of conventional therapy, Xuezhikang showed no significant difference in the number of rehospitalization (RR 1.02; 95\% CI 0.57 to 1.84; 1 trial, $n=84$ ) [28]. Compared with no treatment on the basis of simvastatin and conventional therapy, Xuezhikang showed no effect in reducing rehospitalization (RR $0.20 ; 95 \%$ CI 0.03 to $1.59 ; 1$ trial, $n=48$ ) [27].

3.3.6. Serum TC Level. There were 21 studies that reported the level of total cholesterol Table 4, but one trial only reported the serum lipid level of the treatment group [30]. (1) Compared to no treatment with cointervention of conventional therapy, Xuezhikang showed a reduction of TC level $(\mathrm{MD}-0.97 \mathrm{mmol} / \mathrm{L} ; 95 \% \mathrm{CI}-1.24$ to $-0.71 ; 8$ trials, $n=$ 500) $[5,6,24,29,33,34,38,39]$. (2) There were two trials that reported Xuezhikang versus placebo on the basis of conventional therapy, meta-analysis was not used for significant difference, and, in this comparison, Xuezhikang showed a reduction of TC level (MD $-0.57 \mathrm{mmol} / \mathrm{L} ; 95 \% \mathrm{CI}-0.61$ to -0.53 ; 1 trial, $n=4870$ ) [4] and (MD $-2.62 \mathrm{mmol} / \mathrm{L} ; 95 \%$ CI -2.98 to -2.26 ; 1 trial, $n=62$ ) [35]. (3) There was no significant difference on serum TC level of Xuezhikang comparing to statins on the basis of conventional therapy (MD $0.19 \mathrm{mmol} / \mathrm{L} ; 95 \% \mathrm{CI}-0.22$ to 0.59 ; 8 trial, $n=633$ ) [21, 23$25,28,31,37,39]$. Since there was significant heterogeneity in the comparison, we examined the data carefully and found that data of two trials deviated from the others. After looking over the papers, one of the two trial [26] with an unclear conventional therapy and the other used Xuezhikang $300 \mathrm{mg}$ tid in the whole trial [37]. Sensitive analysis was used and got a similar conclusion (MD $0.02 \mathrm{mmol} / \mathrm{L} ; 95 \% \mathrm{CI}-0.03$ to 0.06; 6 trial, $n=489$ ) after excluded the two trials $[26,37]$. (4) Compared with no treatment on the basis of statins and conventional therapy, Xuezhikang showed a reduction of TC level (MD $-0.96 \mathrm{mmol} / \mathrm{L} ; 95 \% \mathrm{CI}-1.33$ to $-0.58 ; 2$ trial, $n=108)[27,36]$. (5) Compared to inositol nicotinate on the basis of aspirin, Xuezhikang showed a significant difference in the reduction of TC level (MD $-1.05 \mathrm{mmol} / \mathrm{L}$; $95 \%$ CI -1.46 to $-0.64 ; 1$ trial, $n=105$ ) [32].

3.3.7. Serum TG Level. There were 20 studies that reported the level of TG (see Table 4), but one trial only reported the serum lipid level of the treatment group [30]. (1) Compared to no treatment with cointervention of conventional therapy, Xuezhikang showed a reduction of TG level $(\mathrm{MD}-0.49 \mathrm{mmol} / \mathrm{L} ; 95 \% \mathrm{CI}-0.58$ to $-0.39 ; 7$ trial, $n=$ 412) $[5,6,24,29,33,38,39]$. (2) There were two trials that reported Xuezhikang versus placebo on the basis of conventional therapy, meta-analysis was not used for significant difference, and, in this comparison, Xuezhikang showed a reduction of TG level (MD $-0.17 \mathrm{mmol} / \mathrm{L} ; 95 \% \mathrm{CI}-0.22$ to -0.12 ; 1 trial, $n=4870$ ) [4] and (MD $-1.29 \mathrm{mmol} / \mathrm{L} ; 95 \%$ CI -1.57 to $-1.01 ; 1$ trial, $n=62$ ) [35]. (3) There was no significant difference on serum TG level of Xuezhikang comparing to statins on the basis of conventional therapy (MD $-0.05 \mathrm{mmol} / \mathrm{L} ; 95 \% \mathrm{CI}-0.12$ to $0.02 ; 8$ trial, $n=633$ ) [21, 23-25, 28, 31, 37, 39]. (4) Compared with no treatment on the basis of fluvastatin and conventional therapy, Xuezhikang showed a reduction of TG level ( $\mathrm{MD}-0.27 \mathrm{mmol} / \mathrm{L} ; 95 \% \mathrm{CI}$ -0.35 to $-0.19 ; 1$ trial, $n=60$ ) [36]. (5) Compared to inositol nicotinate on the basis of aspirin, Xuezhikang showed a significant difference in the reduction of TG level (MD $-0.60 \mathrm{mmol} / \mathrm{L} ; 95 \% \mathrm{CI}-0.95$ to $-0.25 ; 1$ trial, $n=105$ ) [32].

3.3.8. Serum $L D L-C$ Level. There were 21 studies that reported the level of LDL-C (see Table 4), but one trial only reported the serum lipid level of the treatment group [30]. (1) Compared to no treatment with cointervention of conventional therapy, Xuezhikang showed a reduction of LDL-C level (MD $-0.78 \mathrm{mmol} / \mathrm{L} ; 95 \% \mathrm{CI}-1.19$ to $-0.38 ; 7$ trial, $n=444)[5,6,24,33,34,38,39]$. (2) There were two trials that reported Xuezhikang versus placebo on the basis of conventional therapy, meta-analysis was not used for significant difference, and, in this comparison, Xuezhikang showed a reduction of LDL-C level (MD $-0.57 \mathrm{mmol} / \mathrm{L} ; 95 \% \mathrm{CI}-0.62$ to $-0.52 ; 1$ trial, $n=4870)[4]$ and $(\mathrm{MD}-1.82 \mathrm{mmol} / \mathrm{L} ; 95 \%$ CI -2.01 to $-1.63 ; 1$ trial, $n=62$ ) [35]. (3) There was no significant difference on serum LDL-C level of Xuezhikang comparing to statins on the basis of conventional therapy (MD $0.03 \mathrm{mmol} / \mathrm{L} ; 95 \% \mathrm{CI}-0.10$ to $0.25 ; 8$ trial, $n=633$ ) $[21,23-25,28,31,37,39]$. Because there was significant heterogeneity in the comparison, we examined the data carefully and found that data of two trials deviated from the others. After looking over the papers, one of the two trials [26] with an unclear conventional therapy and the other used Xuezhikang $300 \mathrm{mg}$ tid in the whole trial [37]. 


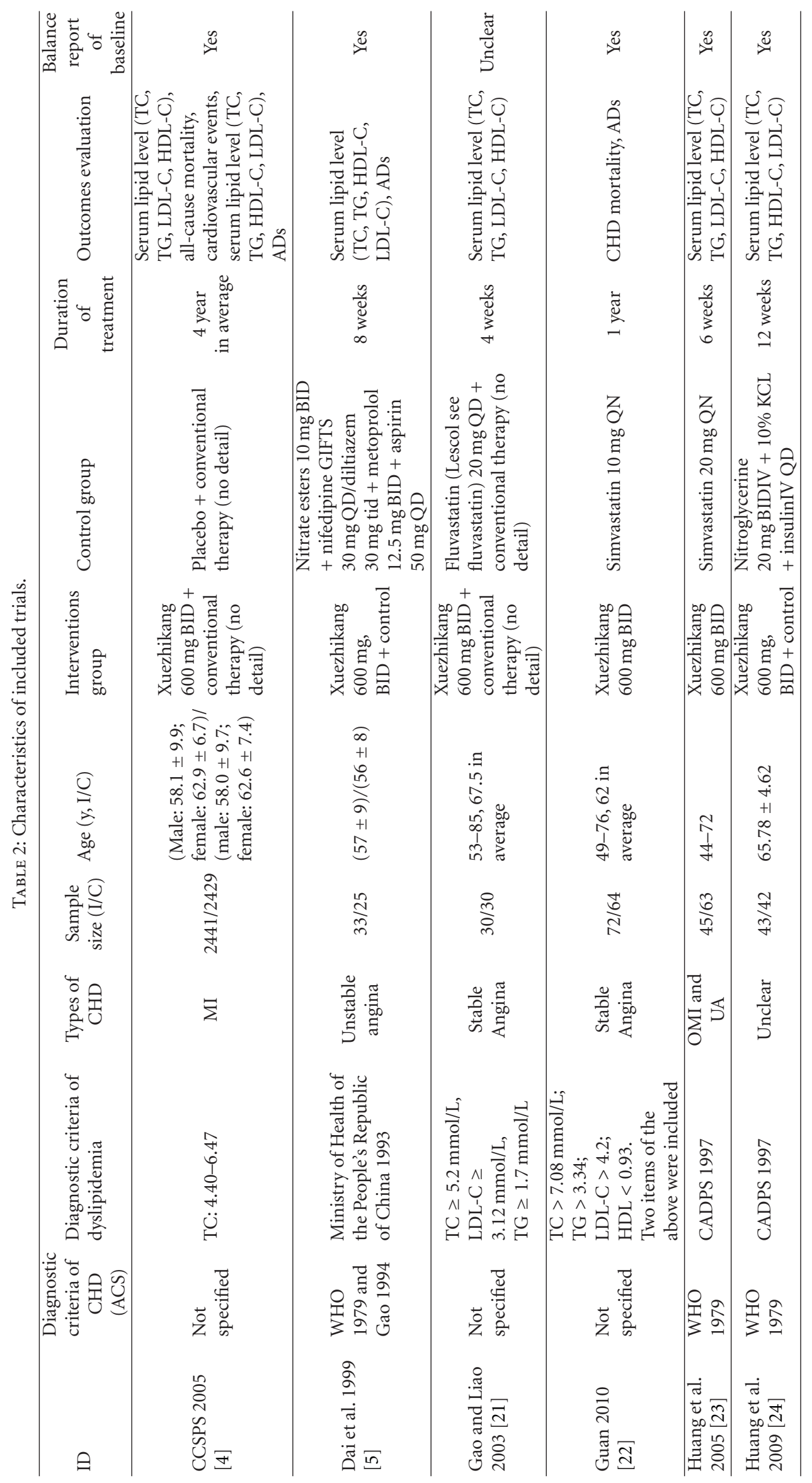




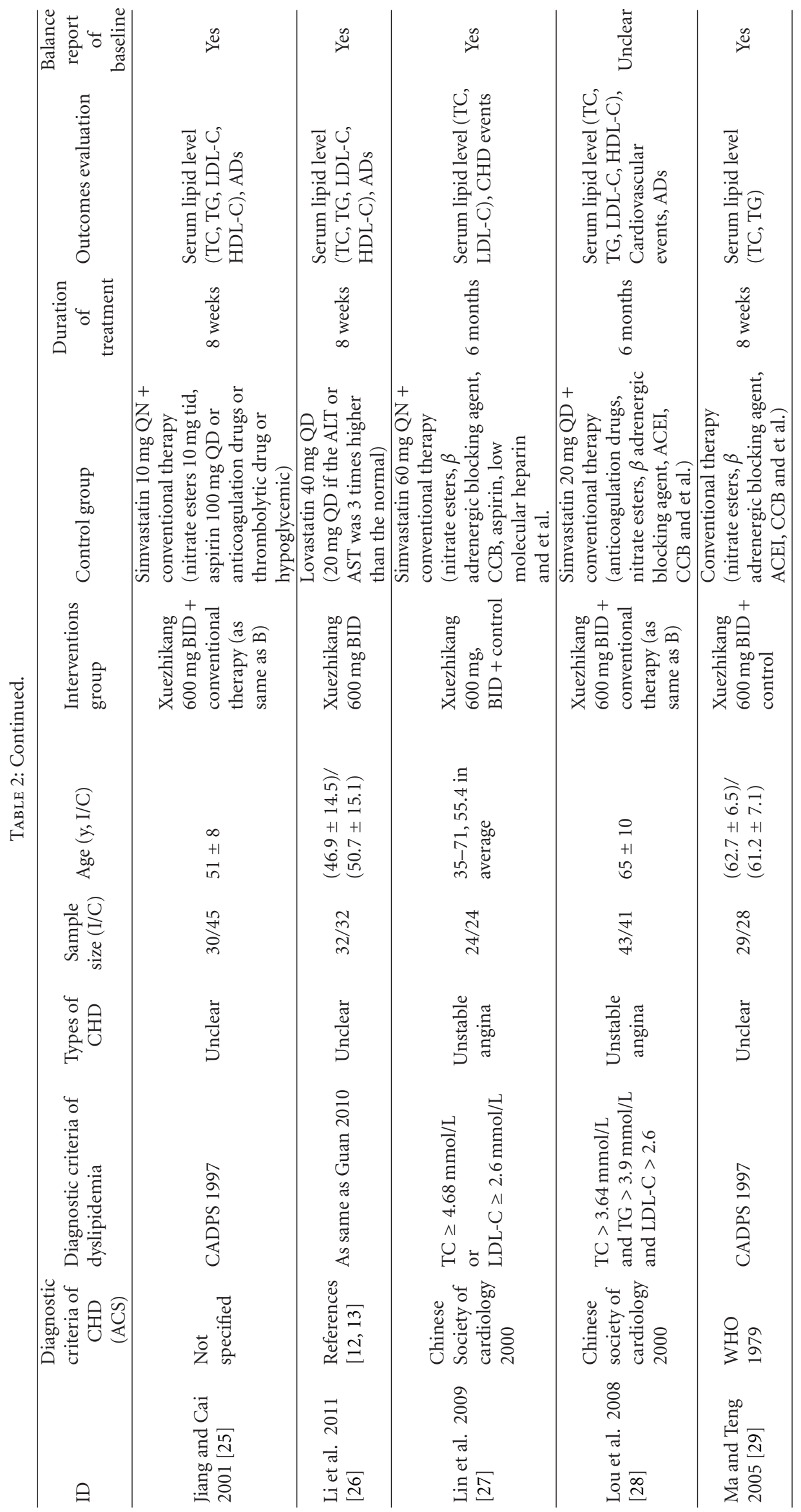




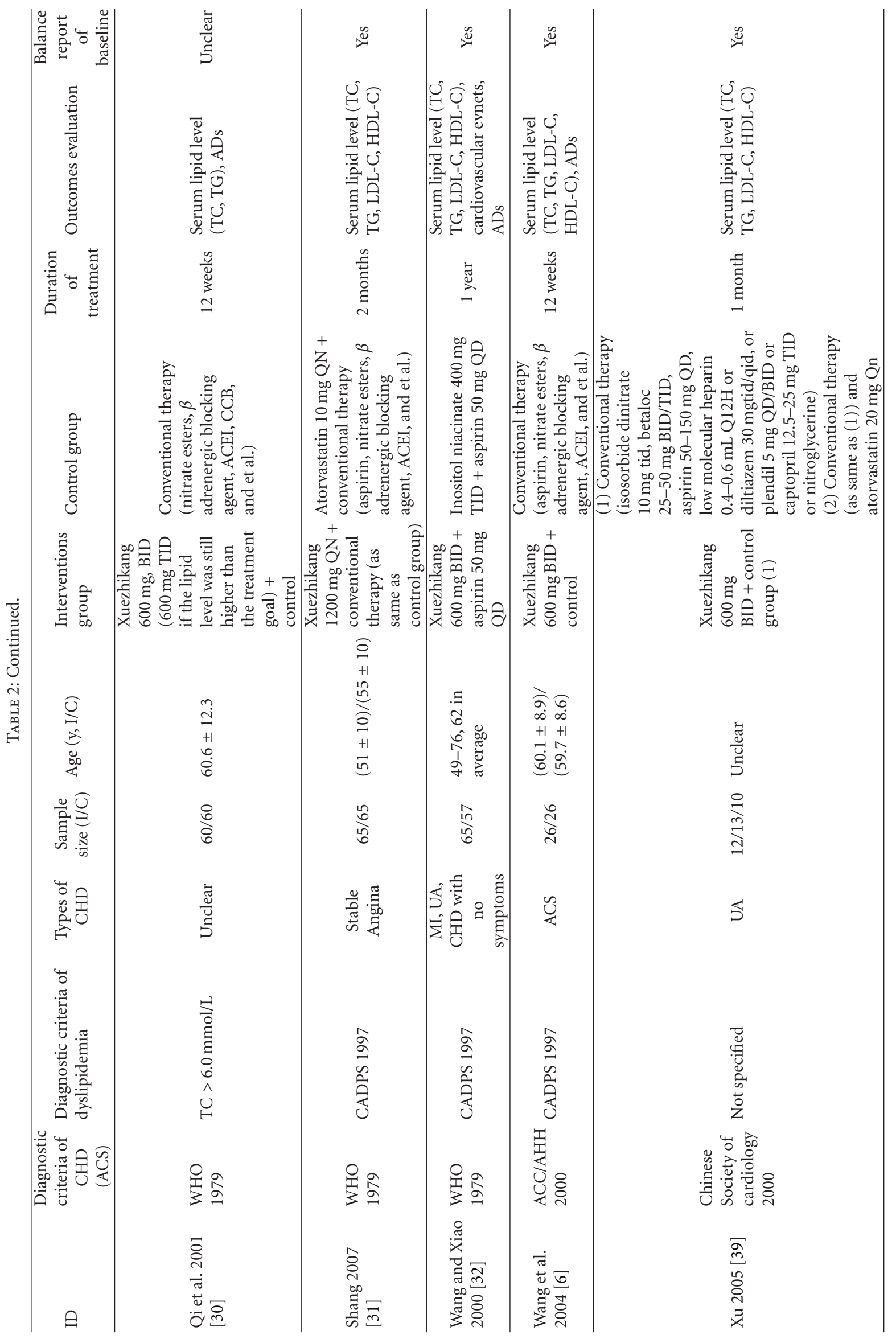




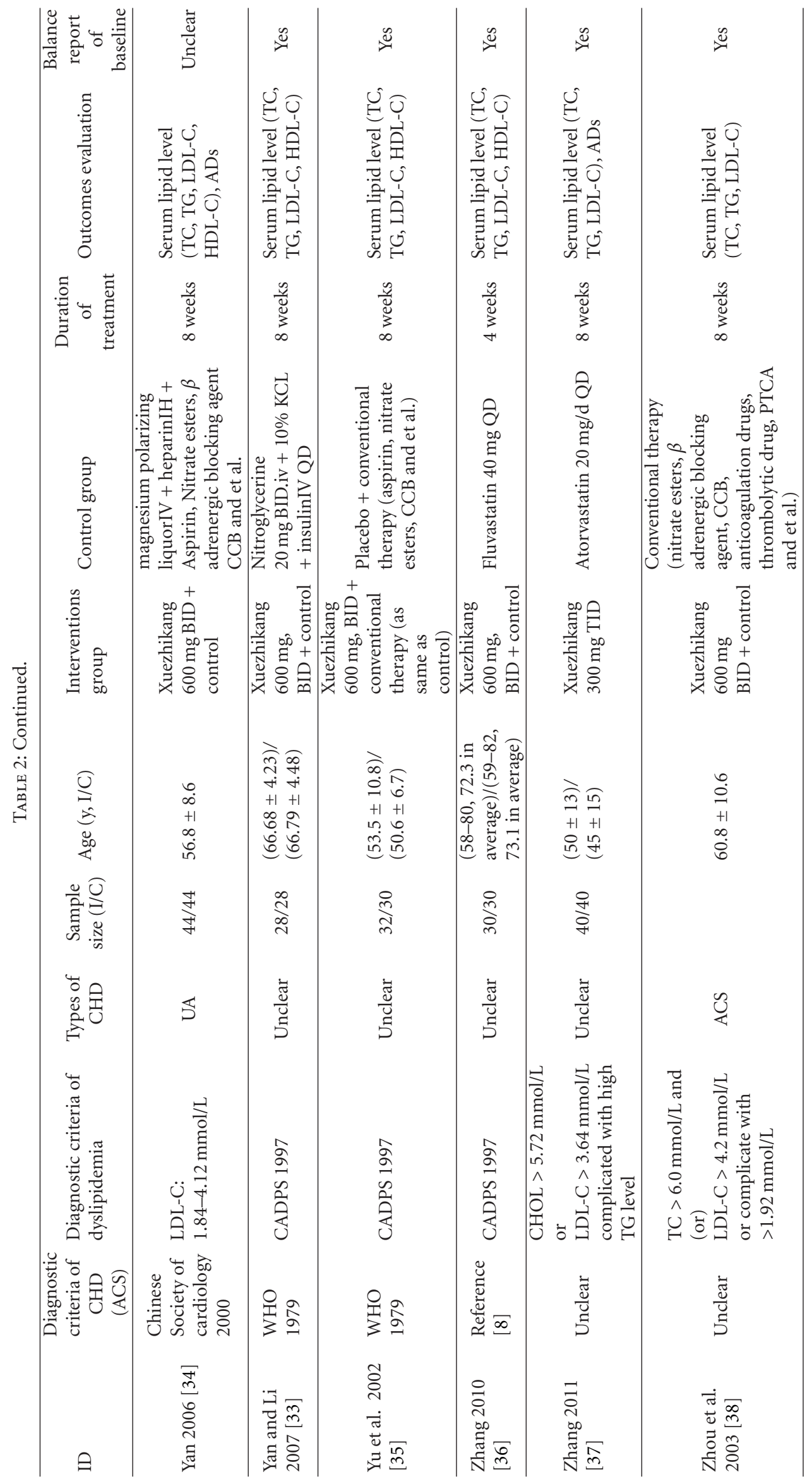


TABLe 3: Analysis of clinical events.

Outcomes (comparisons)

Treatment

Control group

$\operatorname{group}(n / N)$

$(n / N)$

(1) All-cause mortality

Xuezhikang capsule and conventional therapy versus placebo and conventional therapy

CCSPS 2005 [4]

$126 / 2429$

$189 / 2441$

0.67

$[0.54,0.83]$

(2) Mortality of CHD

(2.1) Xuezhikang capsule and conventional therapy versus placebo and conventional therapy

CCSPS 2005 [4] $92 / 2429 \quad 134 / 2441$
$(2.2)$ Xuezhikang and conventional therapy versus simvastatin and conventional therapy

$\begin{array}{llccc}\text { Guan } 2010[22] & 1 / 72 & 6 / 64 & 0.15 & {[0.02,1.20]} \\ \text { Lou et al. 2008 [28] } & 1 / 43 & 1 / 41 & 0.95 & {[0.06,14.75]} \\ & \left.\text { Overall (FEM, } I^{2}=13 \%\right) & \mathbf{0 . 2 6} & {[\mathbf{0 . 0 6}, \mathbf{1 . 2 1}]}\end{array}$

(2.3) Xuezhikang and simvastatin and conventional therapy versus simvastatin and conventional therapy

Lin et al. 2009 [27]

$0 / 24 \quad 1 / 24$

0.33

$[0.01,7.8]$

(2.4) Xuezhikang and aspirin versus inositol nicotinate and aspirin

Wang and Xiao 2000 [32]

$1 / 65$

$6 / 57$

0.15

$[0.02,1.18]$

(3) Myocardial infarction

(3.1) Xuezhikang and conventional therapy versus placebo and conventional therapy
CCSPS 2005 [4]
$47 / 2429$
$120 / 2441$

0.39

$[0.28,0.55]$

(3.2) Xuezhikang and conventional therapy versus simvastatin and conventional therapy

Lou et al. 2008 [28] $\quad 5 / 43 \quad 5 / 41 \quad 0.95$

(3.3) Xuezhikang and simvastatin and conventional therapy versus simvastatin and conventional therapy

Lin et al. 2009 [27] $\quad 0 / 24 \quad 2 / 24 \quad 0.2$

(4) Revascularization

(4.1) Xuezhikang capsule and conventional therapy versus placebo and conventional therapy

CCSPS 2005 [4]

$73 / 2429$

$110 / 2441$

0.67

$[0.50,0.895]$

(4.2) Xuezhikang and conventional therapy versus simvastatin and conventional therapy

Lou et al. 2008 [28]

$6 / 43$

$5 / 41$

1.14

$[0.38,3.46]$

(5) Rehospitalization

(5.1) Xuezhikang and conventional therapy versus simvastatin and conventional therapy

Lou et al 2008 [28] $\quad 15 / 43 \quad 14 / 41 \quad 1.02$

$[0.57,1.84]$

(5.2) Xuezhikang and simvastatin and conventional therapy versus simvastatin and conventional therapy

Lin et al. 2009 [27]

$1 / 24$

$5 / 24$

0.2

$[0.03,1.59]$

Sensitive analysis was used and got a similar conclusion (MD $0.05 \mathrm{mmol} / \mathrm{L} ; 95 \% \mathrm{CI}-0.09$ to $0.19 ; 6$ trial, $n=489$ ) after excluded the two trials [26, 37]. (4) Compared with no treatment on the basis of statins and conventional therapy, Xuezhikang showed a reduction of LDL-C level $(\mathrm{MD}-0.44 \mathrm{mmol} / \mathrm{L} ; 95 \% \mathrm{CI}-0.57$ to $-0.31 ; 2$ trial, $n=$ 108) $[27,36]$. (5) Compared to inositol nicotinate on the basis of aspirin, Xuezhikang showed a significant difference in the reduction of LDL-C level (MD $-0.88 \mathrm{mmol} / \mathrm{L}$; $95 \% \mathrm{CI}$ -1.27 to -0.48 ; 1 trial, $n=105$ ) [32].

3.3.9. Serum HDL-C Level. There were 19 studies that reported the level of HDL-C (see Table 4), but one trial only reported the serum lipid level of the treatment group [30]. (1) Compared to no treatment with cointervention of conventional therapy, Xuezhikang showed a beneficial effect of HDL-C level (MD $0.24 \mathrm{mmol} / \mathrm{L} ; 95 \%$ CI 0.08 to $0.40 ; 6$ trial, $n=364)[5,6,24,33,34,39]$. (2) There were two trials that reported Xuezhikang versus placebo on the basis of conventional therapy, meta-analysis was not used for significant difference, and, in this comparison, Xuezhikang showed a beneficial effect of HDL-C level (MD $0.05 \mathrm{mmol} / \mathrm{L}$; $95 \%$ CI 0.03 to $0.07 ; 1$ trial, $n=4870)$ [4] and (MD $0.48 \mathrm{mmol} / \mathrm{L} ; 95 \%$ CI 0.37 to $0.59 ; 1$ trial, $n=62$ ) [35]. (3) There was a lower effect on serum HDL-C level of Xuezhikang comparing to statins on the basis of conventional therapy (MD $-0.10 \mathrm{mmol} / \mathrm{L} ; 95 \% \mathrm{CI}-0.19$ to $-0.01 ; 8$ trial, $n=633)[21,23-25,28,31,37,39]$. Because there was significant heterogeneity in the comparison, we examined the data carefully and found that data of one trials deviated from the others. After looking over the papers, we found that the trial used Xuezhikang $300 \mathrm{mg}$ tid [37]. Sensitive analysis was used and got a similar conclusion $(\mathrm{MD}-0.10 \mathrm{mmol} / \mathrm{L}$; 95\% CI -0.11 to -0.08 ; 7 trial, $n=553$ ) after excluded the trial [37]. (4) Compared with no treatment on the basis of 
TABLE 4: Analysis of serum lipid level.

\begin{tabular}{lcccc}
\hline \multirow{2}{*}{$\begin{array}{l}\text { Serum lipid level } \\
\text { (comparison) }\end{array}$} & \multicolumn{2}{c}{ Intervention group } & \multicolumn{2}{c}{ Control group } \\
& Mean & SD & Mean & SD \\
\hline (1) TC (mmol/L) & \multicolumn{5}{c}{} \\
(1.1) Xuezhikang and conventional therapy versus conventional therapy \\
Dai et al. 1999 [5] & 5.41 & 0.87 & 6.54 & 0.89 \\
Huang et al. 2009 [24] & 4.98 & 0.79 & 5.99 & 0.87 \\
Ma and Teng 2005 [29] & 5.30 & 1.30 & 6.30 & 1.00 \\
Wang et al. 2004 [6] & 4.33 & 0.96 & 6.30 & 0.79 \\
Xu 2005 [39] & 5.49 & 1.12 & 6.20 & 0.93 \\
Yan 2006 [34] & 4.90 & 0.10 & 5.50 & 0.20 \\
Yan and Li 2007 [33] & 4.90 & 0.13 & 5.93 & 0.23 \\
Zhou et al. 2003 [38] & 4.30 & 0.54 & 4.84 & 0.78 \\
& & \multicolumn{3}{c}{ Overall (REM, $\left.I^{2}=92 \%\right)$}
\end{tabular}

(1.2) Xuezhikang and conventional therapy versus placebo and conventional therapy

$\begin{array}{lllll}\text { CCSPS 2005 [4] } & 4.65 & 0.67 & 5.22 & 0.88 \\ \text { Yu et al. 2002 [35] } & 4.10 & 0.58 & 6.72 & 0.85\end{array}$

(1.3) Xuezhikang and conventional therapy versus statin and conventional therapy

(1.3.1) Xuezhikang and conventional therapy versus lovastatin and conventional therapy

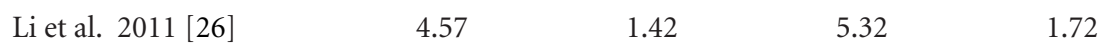

(1.3.2) Xuezhikang and conventional therapy versus simvastatin and conventional therapy

\begin{tabular}{lcccc} 
Huang et al. 2005 [23] & 4.62 & 0.63 & 4.36 & 0.60 \\
Jiang and Cai 2001 [25] & 5.19 & 0.90 & 4.91 & 0.66 \\
Lou et al. 2008 [28] & 5.4 & 0.12 & 5.40 & 0.11 \\
& Subgroup & \multicolumn{3}{c}{ Overall (REM, $\left.I^{2}=69 \%\right)$}
\end{tabular}

(1.3.3) Xuezhikang and conventional therapy versus fluvastatin and conventional therapy
Gao and Liao 2003 [21]
4.05
0.74
3.63
0.59

(1.3.4) Xuezhikang and conventional therapy versus atorvastatin and conventional therapy

\begin{tabular}{lcccc} 
Shang 2007 [31] & 4.65 & 0.79 & 4.88 & 0.85 \\
Xu 2005 [39] & 5.49 & 1.12 & 5.50 & 0.92 \\
Zhang 2011 [37] & 4.51 & 0.38 & 4.00 & 3.35 \\
& Subgroup & \multicolumn{3}{c}{ Overall (REM, $\left.I^{2}=97 \%\right)$} \\
After sensitive analysis & Subgroup & \multicolumn{3}{c}{ Overall (FEM, $\left.I^{2}=0 \%\right)$} \\
& Total & Overall (REM, $\left.I^{2}=96 \%\right)$ & Overall (REM, $\left.I^{2}=66 \%\right)$
\end{tabular}

(1.4) Xuezhikang and statin and conventional therapy versus statin and conventional therapy

(1.4.1) Xuezhikang and simvastatin and conventional therapy versus simvastatin and conventional therapy

$\begin{array}{lllll}\text { Lin et al. } 2009 \text { [27] } & 4.30 & 0.71 & 5.00 & 0.81\end{array}$

(1.4.2) Xuezhikang and fluvastatin and conventional therapy versus fluvastatin and conventional therapy
Zhang 2010 [36]

4.60
0.10
5.70
0.24
Total
Overall $\left(\mathrm{REM}, I^{2}=68 \%\right)$
64.4
(1.5) Xuezhikang and aspirin versus inositol nicotinate and aspirin
$\begin{array}{lllll}\text { Wang and Xiao } 2000[32] & 5.20 & 0.80 & 6.00 & 0.70\end{array}$
0.70

2. $T G(\mathrm{mmol} / \mathrm{L})$

(2.1) Xuezhikang and conventional therapy versus conventional therapy

\begin{tabular}{lllllllr} 
Dai et al. 1999 [5] & 1.84 & 0.68 & 2.30 & 0.87 & 5.50 & -0.48 & {$[-0.87,-0.05]$} \\
Huang et al. 2009 [24] & 1.49 & 0.31 & 1.97 & 0.37 & 44.40 & -0.48 & {$[-0.63,-0.33]$} \\
Ma and Teng 2005 [29] & 1.70 & 0.40 & 2.30 & 0.70 & 10.50 & -0.60 & {$[-0.90,-0.30]$} \\
\hline
\end{tabular}


TABle 4: Continued.

\begin{tabular}{lcccc}
\hline $\begin{array}{l}\text { Serum lipid level } \\
\text { (comparison) }\end{array}$ & \multicolumn{2}{c}{ Intervention group } & \multicolumn{2}{c}{ Control group } \\
& Mean & SD & Mean & SD \\
Wang et al. 2004 [6] & 1.88 & 0.5 & 2.2 & 0.76 \\
Xu 2005 [39] & 2.70 & 0.92 & 2.52 & 1.67 \\
Yan and Li 2007 [33] & 1.54 & 0.10 & 2.02 & 0.59 \\
Zhou et al. 2003 [38] & 1.20 & 0.66 & 1.80 & 0.61 \\
& \multicolumn{4}{c}{ Overall (FEM, $\left.I^{2}=0 \%\right)$} \\
(2.2) Xuezhikang and conventional therapy versus placebo and conventional therapy \\
CCSPS 2005 [4] & 1.58 & 0.78 & 1.75 & 0.88 \\
Yu et al. 2002 [35] & 2.22 & 0.71 & 3.51 & 0.36 \\
(2.3) Xuezhikang and conventional therapy versus statin and conventional therapy \\
(2.3.1) Xuezhikang and conventional therapy versus lovastatin and conventional therapy \\
Li et al. 2011 [26] & 3.75 & 1.17 & 3.82 & 1.29 \\
(2.3.2) Xuezhikang and conventional therapy versus simvastatin and conventional therapy \\
Huang et al. 2005 [23] & 1.85 & 0.81 & 1.92 & 0.72 \\
Jiang and Cai 2001 [25] & 1.9 & 0.72 & 2.11 \\
Lou et al. 2008 [28] & 3.1 & 0.2 & 3.2 & 0.91 \\
& Subgroup & Overall (FEM, $\left.I^{2}=0 \%\right)$ &
\end{tabular}

(2.3.3) Xuezhikang and conventional therapy versus fluvastatin and conventional therapy
Gao and Liao 2003 [21]
1.01
0.63
1.42
0.46

(2.3.4) Xuezhikang and conventional therapy versus atorvastatin and conventional therapy

$\begin{array}{lccc}\text { Shang } 2007 \text { [31] } & 1.61 & 0.53 & 1.57 \\ \text { Xu 2005 [39] } & 2.7 & 0.92 & 2.22 \\ \text { Zhang 2011 [37] } & 1.64 & 0.33 & 1.61 \\ & \text { Subgroup } & & \left.\text { Overall (FEM, } I^{2}=0 \%\right) \\ & \text { Total } & & \left.\text { Overall (FEM, } I^{2}=45 \%\right)\end{array}$

0.55

0.73

0.21

Total Overall (FEM, $\left.I^{2}=45 \%\right)$

(2.4) Xuezhikang and statin and conventional therapy versus statin and conventional therapy
Zhang 2010 [36]
1.58
0.20
1.85
0.10

(2.5) Xuezhikang and aspirin versus inositol nicotinate and aspirin

Wang and Xiao 2000 [32] $\quad 1.70 \quad 0.90$

$2.30 \quad 0.90$

(3) $\mathrm{LDL}-\mathrm{C}(\mathrm{mmol} / \mathrm{L})$

(3.1) Xuezhikang and conventional therapy versus conventional therapy

$\begin{array}{lcccc}\text { Dai et al. 1999 [5] } & 3.42 & 0.96 & 3.93 & 0.81 \\ \text { Huang et al. 2009 [24] } & 2.88 & 0.91 & 3.96 & 0.96 \\ \text { Wang et al. 2004 [6] } & 2.21 & 0.4 & 3.87 & 0.56 \\ \text { Xu 2005 [39] } & 2.82 & 0.95 & 3.7 & 0.95 \\ \text { Yan 2006 [34] } & 2.89 & 0.44 & 2.9 & 0.6 \\ \text { Yan and Li 2007 [33] } & 2.97 & 0.10 & 3.88 & 0.20 \\ \text { Zhou et al. 2003 [38] } & 3.22 & 0.6 & 3.68 & 0.71\end{array}$

(3.2) Xuezhikang and conventional therapy versus placebo and conventional therapy

$\begin{array}{lllll}\text { CCSPS 2005 [4] } & 2.66 & 0.85 & 3.23 & 0.85 \\ \text { Yu et al. 2002 [35] } & 2.48 & 0.39 & 4.30 & 0.39\end{array}$

(3.3) Xuezhikang and conventional therapy versus statin and conventional therapy 0.39 (3.3.1) Xuezhikang and conventional therapy versus lovastatin and conventional therapy

50.80

49.20

$$
\begin{array}{ll}
-0.17 & {[-0.22,-0.12]} \\
-1.29 & {[-1.57,-1.01]}
\end{array}
$$

\begin{tabular}{ccc}
1.3 & -0.07 & {$[-0.67,0.53]$} \\
5.5 & -0.07 & {$[-0.37,0.23]$} \\
3.5 & -0.21 & {$[-0.58,0.16]$} \\
35.2 & -0.11 & {$[-0.21,0.00]$} \\
$\mathbf{4 4 . 3}$ & $\mathbf{0 . 1 1}$ & {$[-\mathbf{0 . 2 1},-\mathbf{0 . 0 0}]$} \\
$\mathbf{6 . 2}$ & -0.41 & {$[-0.69,-0.13]$} \\
$\mathbf{1 4 . 1}$ & 0.04 & {$[-0.15,0.23]$} \\
1.0 & 0.48 & {$[-0.21,1.17]$} \\
33.0 & 0.03 & {$[-0.09,0.15]$} \\
$\mathbf{4 8 . 1}$ & $\mathbf{0 . 0 4}$ & {$[-\mathbf{0 . 0 6}, \mathbf{0 . 1 4}]$} \\
$\mathbf{1 0 0}$ & $-\mathbf{0 . 0 5}$ & {$[-\mathbf{0 . 1 2}, \mathbf{0 . 0 2}]$} \\
& & \\
\hline & -0.27 & {$[-0.35,-0.19]$} \\
& & \\
\hline & -0.60 & {$[-0.95,-0.25]$}
\end{tabular}

$\begin{array}{ccc}13.50 & -0.51 & {[-0.97,-0.05]} \\ 14.10 & -1.08 & {[-1.48,-0.68]} \\ 15.20 & -1.66 & {[-1.92,-1.40]} \\ 10.50 & -0.88 & {[-1.63,-0.13]} \\ 15.50 & -0.01 & {[-0.23,0.21]} \\ 16.20 & -0.91 & {[-0.99,-0.83]} \\ 15.00 & -0.46 & {[-0.75,-0.17]} \\ 100 & -0.78 & {[-1.19,-0.38]} \\ & & \\ 50.30 & -0.57 & {[-0.62,-0.52]} \\ 49.70 & -1.82 & {[-2.01,-1.63]}\end{array}$


Table 4: Continued.

\begin{tabular}{|c|c|c|c|c|c|c|c|}
\hline \multirow{2}{*}{$\begin{array}{l}\text { Serum lipid level } \\
\text { (comparison) }\end{array}$} & \multicolumn{2}{|c|}{ Intervention group } & \multicolumn{2}{|c|}{ Control group } & \multirow{2}{*}{ Weight $(\%)$} & \multirow{2}{*}{ MD } & \multirow{2}{*}{$95 \% \mathrm{CI}$} \\
\hline & Mean & SD & Mean & $\mathrm{SD}$ & & & \\
\hline \multicolumn{8}{|c|}{ (3.3.2) Xuezhikang and conventional therapy versus simvastatin and conventional therapy } \\
\hline Huang et al. 2005 [23] & 2.68 & 0.55 & 2.52 & 0.49 & 13.9 & 0.16 & {$[-0.04,0.36]$} \\
\hline Jiang and Cai $2001[25]$ & 3.1 & 0.41 & 2.90 & 0.90 & 12.2 & 0.20 & {$[-0.10,0.50]$} \\
\hline \multirow[t]{2}{*}{ Lou et al. 2008 [28] } & 2.8 & 0.09 & 2.9 & 0.1 & 15.7 & -0.10 & {$[-0.14,-0.06]$} \\
\hline & Subtotal & & (REM, 1 & & 41.8 & 0.06 & {$[-0.17,0.28]$} \\
\hline \multicolumn{8}{|c|}{ (3.3.3) Xuezhikang and conventional therapy versus fluvastatin and conventional therapy } \\
\hline Gao and Liao 2003 [21] & 2.13 & 0.58 & 2.08 & 0.61 & 12.2 & 0.05 & {$[-0.25,0.35]$} \\
\hline \multicolumn{8}{|c|}{ (3.3.4) Xuezhikang and conventional therapy versus atorvastatin and conventional therapy } \\
\hline Shang 2007 [31] & 2.54 & 0.56 & 2.44 & 0.52 & 14.2 & 0.10 & {$[-0.09,0.29]$} \\
\hline Xu 2005 [39] & 2.82 & 0.95 & 2.93 & 0.52 & 6.9 & -0.11 & {$[-0.74,0.52]$} \\
\hline \multirow[t]{2}{*}{ Zhang 2011 [37] } & 3.04 & 0.48 & 2.51 & 0.32 & 14.3 & 0.53 & {$[0.35,0.71]$} \\
\hline & Subtotal & & REM, I & & 35.4 & 0.23 & {$[-0.14,0.60]$} \\
\hline \multirow[t]{2}{*}{ After sensitive analysis } & Subtotal & & (FEM, & & & 0.08 & {$[-0.10,0.26]$} \\
\hline & Total & & REM, $I$ & & & 0.03 & {$[-0.10,0.25]$} \\
\hline After sensitive analysis & Total & & REM, I & & & 0.05 & {$[-0.09,0.19]$} \\
\hline \multicolumn{8}{|c|}{ (3.4) Xuezhikang and statin and conventional therapy versus statin and conventional therapy } \\
\hline \multicolumn{8}{|c|}{ (3.4.1) Xuezhikang and simvastatin and conventional therapy versus simvastatin and conventional therapy } \\
\hline Lin et al. 2009 [27] & 2.10 & 0.78 & 2.60 & 0.80 & 8.4 & -0.50 & {$[-0.95,-0.05]$} \\
\hline \multicolumn{8}{|c|}{ (3.4.2) Xuezhikang and fluvastatin and conventional therapy versus fluvastatin and conventional therapy } \\
\hline \multirow[t]{2}{*}{ Zhang $2010[36]$} & 2.87 & 0.32 & 3.30 & 0.20 & 91.6 & -0.43 & {$[-0.57,-0.29]$} \\
\hline & Total & & (FEM, & & & -0.44 & {$[-0.57,-0.31]$} \\
\hline \multicolumn{8}{|c|}{ (3.5) Xuezhikang and aspirin versus inositol nicotinate and aspirin } \\
\hline Wang and Xiao $2000[32$ & 2.70 & 0.70 & 3.40 & 0.90 & 100 & -0.88 & {$[-1.27,-0.48]$} \\
\hline \multicolumn{8}{|l|}{ (4) $H D L-C(m m o l / L)$} \\
\hline \multicolumn{8}{|c|}{ (4.1) Xuezhikang and conventional therapy versus conventional therapy } \\
\hline Dai et al. 1999 [5] & 1.71 & 0.42 & 1.04 & 0.49 & 14.60 & 0.67 & {$[-0.43,0.91]$} \\
\hline Huang et al. 2009 [24] & 1.12 & 0.3 & 0.82 & 0.2 & 19.50 & 0.3 & {$[0.19,0.41]$} \\
\hline Wang et al. 2004 [6] & 1.44 & 0.38 & 1.31 & 0.27 & 17.00 & 0.13 & {$[-0.05,0.31]$} \\
\hline Xu 2005 [39] & 1.67 & 0.51 & 1.68 & 0.75 & 7.10 & -0.01 & {$[-0.51,0.49]$} \\
\hline Yan 2006 [34] & 1.04 & 0.10 & 1.04 & 0.20 & 20.60 & 0.00 & {$[-0.07,0.07]$} \\
\hline \multirow[t]{2}{*}{ Yan and Li 2007 [33] } & 1.09 & 0.09 & 0.80 & 0.07 & 21.10 & 0.29 & {$[0.25,0.33]$} \\
\hline & & & REM, 1 & & 100 & 0.24 & {$[0.08,0.40]$} \\
\hline \multicolumn{8}{|c|}{ (4.2) Xuezhikang and conventional therapy versus placebo and conventional therapy } \\
\hline CCSPS 2005 [4] & 1.24 & 0.31 & 1.19 & 0.31 & 50.80 & 0.05 & {$[0.03,0.07]$} \\
\hline Yu et al. 2002 [35] & 1.45 & 0.25 & 0.97 & 0.19 & 49.20 & 0.48 & {$[0.37,0.59]$} \\
\hline \multicolumn{8}{|c|}{ (4.3) Xuezhikang and conventional therapy versus statin and conventional therapy } \\
\hline (4.3.1) Xuezhikang and co & ational the & sus lov & d conve & erapy & & & \\
\hline Li et al. $2011[26]$ & 1.12 & 0.38 & 1.06 & 0.36 & 11.4 & 0.16 & {$[-0.33,0.65]$} \\
\hline (4.3.2) Xuezhikang and co & ntional the & sus sim & nd con & herapy & & & \\
\hline Huang et al. 2005 [23] & 1.85 & 0.81 & 1.92 & 0.72 & 6.4 & -0.09 & {$[-0.47,0.29]$} \\
\hline Jiang and Cai 2001 [25] & 1.16 & 0.17 & 1.21 & 0.12 & 19.0 & -0.05 & {$[-0.12,0.02]$} \\
\hline Lou et al. 2008 [28] & 0.8 & 0.03 & 0.9 & 0.03 & 21.4 & -0.10 & {$[-0.11,-0.09]$} \\
\hline & & & (FEM, & & & -0.10 & {$[-0.11,-0.09]$} \\
\hline (4.3.3) Xuezhikang and co & ntional the & sus flu & id conv & erapy & & & \\
\hline Gao and Liao 2003 [21] & 1.14 & 0.27 & 1.30 & 0.45 & 11 & -0.16 & {$[-0.35,0.03]$} \\
\hline
\end{tabular}


TABle 4: Continued.

\begin{tabular}{|c|c|c|c|c|c|c|c|}
\hline \multirow{2}{*}{$\begin{array}{l}\text { Serum lipid level } \\
\text { (comparison) }\end{array}$} & \multicolumn{2}{|c|}{ Intervention group } & \multicolumn{2}{|c|}{ Control group } & \multirow{2}{*}{ Weight (\%) } & \multirow{2}{*}{ MD } & \multirow{2}{*}{$95 \% \mathrm{CI}$} \\
\hline & Mean & SD & Mean & $\mathrm{SD}$ & & & \\
\hline \multicolumn{8}{|c|}{ (4.3.4) Xuezhikang and conventional therapy versus atorvastatin and conventional therapy } \\
\hline Shang $2007[31]$ & 1.45 & 0.41 & 1.44 & 0.33 & 14.9 & 0.01 & {$[-0.12,0.14]$} \\
\hline Xu 2005 [39] & 1.67 & 0.51 & 1.53 & 0.48 & 3.8 & 0.14 & {$[-0.27,0.55]$} \\
\hline \multirow[t]{2}{*}{ Zhang 2011 [37] } & 1.09 & 0.48 & 1.62 & 0.27 & 12.1 & -0.53 & {$[-0.70,-0.36]$} \\
\hline & Subtotal & & (REM, $I$ & & 30.9 & -0.15 & {$[-0.57,0.28]$} \\
\hline \multirow[t]{2}{*}{ After sensitive analysis } & Subtotal & & $(\mathrm{FEM}$, & & & 0.02 & {$[-0.10,0.14]$} \\
\hline & Total & & (REM, I & & & -0.10 & {$[-0.19,-0.01]$} \\
\hline After sensitive analysis & Total & & (FEM, $I$ & & & -0.10 & {$[-0.11,-0.08]$} \\
\hline \multicolumn{8}{|c|}{ (4.4) Xuezhikang and fluvastatin and conventional therapy versus fluvastatin and conventional therapy } \\
\hline Zhang 2010 [36] & 0.97 & 0.28 & 0.82 & 0.06 & 100 & 0.15 & {$[0.05,0.25]$} \\
\hline \multicolumn{8}{|c|}{ (4.5) Xuezhikang and aspirin versus inositol nicotinate and aspirin } \\
\hline Wang and Xiao 2000 [32] & 0.95 & 0.22 & 0.91 & 0.25 & 100 & 0.17 & {$[-0.21,0.55]$} \\
\hline
\end{tabular}

Note: FEM: fixed effects model; REM: random effects model.

fluvastatin and conventional therapy, Xuezhikang showed a beneficial of HDL-C level (MD $0.15 \mathrm{mmol} / \mathrm{L} ; 95 \%$ CI 0.05 to $0.25 ; 1$ trial, $n=60$ ) [36]. (5) Compared with inositol nicotinate on the basis of aspirin, Xuezhikang showed no significant difference on HDL-C level (MD $0.17 \mathrm{mmol} / \mathrm{L}$; $95 \%$ CI -0.21 to $0.55 ; 1$ trial, $n=105$ ) [32].

3.4. Publication Bias. A funnel plot analysis of the 8 trials in comparison of Xuezhikang and conventional therapy versus conventional therapy on serum TC level was conducted and shown in Figure 3.

3.5. Adverse Events. There were 17 trials that reported adverse events (Ads); see Table 5. 4 of the 17 trials [5, 24, $33,37]$ indicated no Ads in the duration of treatment, and 2 trials $[23,34]$ only introduced that there was no difference of the two groups. The most commonly reported Ads in the 10 trials were intestinal disturbance (abdominal distension, constipation, and diarrhea), dizziness, high serum alanine aminotransferase (ALT), high serum creatine kinase (CK), high serum creatinine, high blood urea nitrogen (BUN), and skin itch. All of Ads were not significantly different between the Xuezhikang group and control group. One trial [4] reported that there was significant difference between the two groups on sexual dysfunction $(P=0.0253)$ in the paper, but after we import the data into Revman 5.1, there was no difference (RR $0.09,95 \%$ CI $[0.01,1.64])$ between the two groups. CCSPS [4] reported the clinical total Ads number (intestinal disturbance, allergy and et al.) in each group (treatment group 43; control group 39), and there was no significant difference between the two groups, this trial also reported death in other reason, which was introduced in allcause mortality, and the difference between the two groups was not significant.

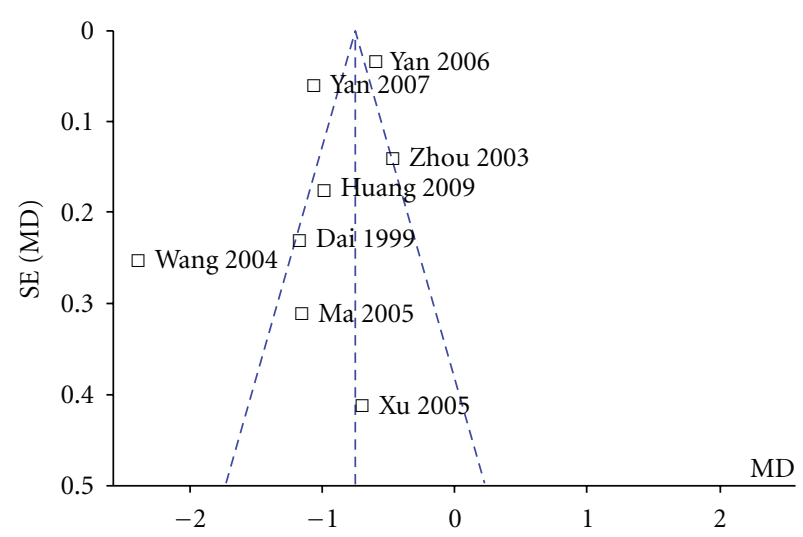

Note: The funnel plot presented 8 trials in the comparison of Xuezhikang and conventional therapy versus conventional therapy on the effect of TC

Figure 3: The funnel plot for assessing reporting bias.

\section{Discussion}

This systematic review included 22 randomized trials and a total of 6520 participants. Xuezhikang showed significant benefit on the incidence of all-cause deaths, CHD deaths, myocardial infarction, and revascularization as compared with placebo or no intervention based on conventional treatment for CHD. It remarkably lowered TC, TG, and LDL-C as compared with the placebo or inositol nicotinate group, which was similar to statins group. Xuezhikang also significantly raised HDL-C compared to placebo or no intervention, which was similar to inositol nicotinate and slightly inferior to statins. The incidence of adverse events did not differ between the Xuezhikang and control group. The results showed the comprehensive lipid-regulating effect of Xuezhikang and indicated that it was safe and effective in reducing cardiovascular events in $\mathrm{CHD}$ patients complicated by dyslipidemia. 
TABle 5: Adverse Events.

\begin{tabular}{|c|c|c|c|c|c|}
\hline Ads/ID & Comparison & $\begin{array}{c}\text { Treatment } \\
\text { group }(n / N)\end{array}$ & $\begin{array}{c}\text { Control } \\
\text { group }(n / N)\end{array}$ & $\mathrm{RR}$ & $95 \% \mathrm{CI}$ \\
\hline \multicolumn{6}{|l|}{ Loss of followup } \\
\hline Guan 2010 [22] & Xuezhikang versus simvastatin & $16(72)$ & $15(64)$ & 0.95 & {$[0.51,1.76]$} \\
\hline CCSPS $2005[4]$ & $\begin{array}{l}\text { Xuezhikang and conventional therapy versus } \\
\text { placebo and conventional therapy }\end{array}$ & $37(2441)$ & $28(2429)$ & 1.31 & {$[0.81,2.14]$} \\
\hline Ma and Teng 2005 [29] & $\begin{array}{l}\text { Xuezhikang and conventional therapy versus } \\
\text { conventional therapy }\end{array}$ & $1(29)$ & No report & & \\
\hline \multicolumn{6}{|l|}{ Intestinal disturbance } \\
\hline Guan 2010 [22] & Xuezhikang versus simvastatin & $5(72)$ & $2(64)$ & 2.22 & {$[0.45,11.06]$} \\
\hline Ma and Teng 2005 [29] & $\begin{array}{l}\text { Xuezhikang and conventional therapy versus } \\
\text { conventional therapy }\end{array}$ & $2(29)$ & No report & & \\
\hline Wang et al. 2004 [6] & $\begin{array}{l}\text { Xuezhikang and conventional therapy versus } \\
\text { conventional therapy }\end{array}$ & $2(26)$ & No report & & \\
\hline Jiang and Cai 2001 [25] & $\begin{array}{l}\text { Xuezhikang and conventional therapy versus } \\
\text { simvastatin and conventional therapy }\end{array}$ & $0(30)$ & $1(45)$ & 0.49 & {$[0.02,11.75]$} \\
\hline Shang 2007 [31] & $\begin{array}{l}\text { Xuezhikang and conventional therapy versus } \\
\text { atorvastatin and conventional therapy }\end{array}$ & No report & $1(65)$ & & \\
\hline Wang and Xiao 2000 [32] & $\begin{array}{l}\text { Xuezhikang and aspirin versus inositol } \\
\text { nicotinate and aspirin }\end{array}$ & $5(65)$ & $2(57)$ & 2.19 & {$[0.44,10.87]$} \\
\hline \multicolumn{6}{|l|}{ Headache } \\
\hline Jiang and Cai 2001 [25] & $\begin{array}{l}\text { Xuezhikang and conventional therapy versus } \\
\text { simvastatin and conventional therapy }\end{array}$ & $1(30)$ & $0(45)$ & 4.45 & {$[0.19,105.77]$} \\
\hline \multicolumn{6}{|l|}{ Dizziness } \\
\hline Guan 2010 [22] & $\begin{array}{l}\text { Xuezhikang and conventional therapy versus } \\
\text { simvastatin and conventional therapy }\end{array}$ & $0(72)$ & $10(64)$ & 0.04 & {$[0.00,0.71]$} \\
\hline \multirow[t]{2}{*}{ Jiang and Cai 2001 [25] } & $\begin{array}{l}\text { Xuezhikang and conventional therapy versus } \\
\text { simvastatin and conventional therapy }\end{array}$ & $1(30)$ & $1(45)$ & 1.5 & {$[0.10,23.07]$} \\
\hline & & \multicolumn{2}{|c|}{ Overall $\left(\mathrm{REM}, I^{2}=72 \%\right)$} & 0.26 & {$[0.01,10.49]$} \\
\hline \multicolumn{6}{|l|}{ Skin itech } \\
\hline Guan 2010 [22] & Xuezhikang versus simvastatin & $0(72)$ & $3(64)$ & 0.13 & {$[0.01,2.42]$} \\
\hline Wang and Xiao 2000 [32] & $\begin{array}{l}\text { Xuezhikang and aspirin versus inositol } \\
\text { nicotinate and aspirin }\end{array}$ & $0(65)$ & $3(57)$ & 0.13 & {$[0.01,2.38]$} \\
\hline \multicolumn{6}{|l|}{ Sexual dysfunction } \\
\hline CCSPS 2005 [4] & $\begin{array}{l}\text { Xuezhikang and conventional therapy versus } \\
\text { placebo and conventional therapy }\end{array}$ & $0(1996)$ & $5(1990)$ & 0.09 & {$[0.01,1.64]$} \\
\hline \multicolumn{6}{|l|}{ High serum ALT } \\
\hline CCSPS 2005 [4] & $\begin{array}{l}\text { Xuezhikang and conventional therapy versus } \\
\text { placebo and conventional therapy }\end{array}$ & $15(2441)$ & $22(2429)$ & 0.68 & {$[0.35,1.30]$} \\
\hline Lou et al. 2008 [28] & $\begin{array}{l}\text { Xuezhikang and conventional therapy versus } \\
\text { simvastatin and conventional therapy }\end{array}$ & No report & $1(41)$ & & \\
\hline \multicolumn{6}{|l|}{ High serum $C K$} \\
\hline CCSPS 2005 [4] & $\begin{array}{l}\text { Xuezhikang and conventional therapy versus } \\
\text { placebo and conventional therapy }\end{array}$ & $0(2441)$ & $3(2429)$ & 0.14 & {$[0.01,2.75]$} \\
\hline \multicolumn{6}{|l|}{ High serum $C R$} \\
\hline CCSPS 2005 [4] & $\begin{array}{l}\text { Xuezhikang and conventional therapy versus } \\
\text { placebo and conventional therapy }\end{array}$ & $104(2441)$ & $89(2429)$ & 1.16 & {$[0.88,1.53]$} \\
\hline \multicolumn{6}{|l|}{ High BUN } \\
\hline CCSPS 2005 [4] & $\begin{array}{l}\text { Xuezhikang and conventional therapy versus } \\
\text { placebo and conventional therapy }\end{array}$ & $124(2441)$ & $131(2429)$ & 0.94 & {$[0.74,1.20]$} \\
\hline
\end{tabular}


Due to the potential side effects of statins, natural products have raised more and more attention worldwide. The health-enhancing qualities of red yeast rice have been introduced and used in China for over two thousand years. A meta-analysis of randomized controlled trials on Chinese red yeast rice for primary hyperlipidemia showed a significant reduction in serum levels of TC, TG, LDL-C, and an increase in HDL-C levels compared with placebo. The lipid modification effects appeared to be similar to pravastatin, simvastatin, atorvastatin, lovastatin, or fluvastatin [40]. A latest systematic review also indicated the beneficial effects of Xuezhikang in the treatment of hyperlipidemia [2]. The lipid-regulating effects of Xuezhikang in these reviews were similar to our findings. In addition, some cardioprotective effects of Xuezhikang have been investigated in recent years [41-43]. We further demonstrated the benefit of Xuezhikang in reducing cardiovascular events in CHD patients complicated by dyslipidemia, or even CHD with normal blood lipid level but failed to reach the lipid-lowering goal. However, current evidence comparing the effectiveness and Ads between Xuezhikang and statins in CHD patients was not enough to draw the conclusion.

It is worth mentioning China Coronary Secondary Prevention Study (CCSPS) [4], which was the largest RCT included in this review. This multicenter, randomized, and placebo-controlled study aimed to demonstrate the longterm therapeutic effect and safety of Xuezhikang in the second prevention of CHD. 4870 cases in 66 medical centers were enrolled and followed up for an average of 4.5 years. The results showed that Xuezhikang significantly decreased the recurrence of coronary events and the occurrence of new cardiovascular events and deaths, improved lipoprotein regulation, and was safe and well tolerated [4]. The study was the first large-scale clinical trial in eastern population who suffered from mild or moderate degree of hyperlipidemia and previous MI. The CCSPS study is quite comparable with (Cholesterol and Recurrent Events) CAREs study [44] in terms of the target population, sample size, baseline lipid and follow-up time. However, Xuezhikang in CCSPS lowered less lipid level as compared with pravastatin in CARE but seemed to gain more benefit in reducing the cardiovascular events. Since the effect of Xuezhikang is partially attributed to the presence of statins, it has been hypothesized that relatively high concentrations of unsaturated fatty acids and other natural compounds found in Xuezhikang may work in concert with the statins to provide additional health benefits [45]. Therefore, a large-scale RCT comparing directly the effectiveness and safety of long-term use of Xuezhikang and statins is warranted.

Before recommending the conclusion of this review to clinical practicers, we have to consider the following weaknesses in this review. (1) Firstly, the "randomization" was not clear in most of the trials for insufficient reporting of generation methods of the allocation sequence and allocation concealment. Most trials stated only that patients were randomly assigned. (2) Secondly, most of trials did not introduce double blind in this review, and only one trial introduced blinding of outcome assessment, therefore, in nonplacebo-controlled and non-double-blind trials, placebo effects may add to the complexity of interpreting the conclusion. (3) Most of the trials did not introduce the study plan, attrition bias and selective reporting bias might exist in this conclusion. (4) Thirdly, funnel plot indicated that publication bias would exist in this review. The reasons are as follows. We only selected trials published in Chinese and English, and trials published in other language or originated from other countries might be omitted; we only identified unpublished studies from conference paper or academic thesis, and negative trials might not be reported and induced publication bias.

Therefore, further rigorously designed trials are still needed before Xuezhikang could be recommended to patients with CHD complicated by dyslipidemia, especially as an alternative to statins. Whether or not long-term medication of Xuezhikang could provide similar benefit to statins for CHD secondary prevention with less adverse events? Is it related to the target lipid value? All of these need to be answered in the future investigation.

\section{Conclusion}

Xuezhikang showed a comprehensive lipid-regulating effect and was safe and effective in reducing CHD mortality, the incidence of myocardial infarction and revascularization in CHD patients complicated by dyslipidemia. However, the small sample size and potential bias of most trials influence the convincingness of this conclusion. Before recommending Xuezhikang as an alternative to statins in CHD patients, more rigorous trials with high quality are needed to give high level of evidence, especially for comparing the effectiveness and safety between Xuezhikang and statins.

\section{Authors' Contributions}

J. Liu and $\mathrm{H} . \mathrm{Xu}$ conceived and designed the review and performed interpretation of the review; Q. Shang, Z. Liu developed the search strategy and did the literature search, study selection, data extraction, data analyses and interpretation; K. Chen provided methodological perspectives and revised review. All of authors contributed to writing the review.

\section{References}

[1] The Task Force for the Management of Dyslipidemias of the European Society of Cardiology (ESC) and the European Atherosclerosis Society (EAS), "ESC/EAS guidelines for the management of dyslipidemias," European Heart Journal, vol. 32, pp. 1769-1818, 2011.

[2] Z. L. Liu, J. P. Liu, A. L. Zhang et al., "Chinese herbal medicines for hypercholesterolemia," Cochrane Database of Systematic Reviews, no. 7, Article ID CD008305, 2011.

[3] Joint Commission on China Adult Dyslipidemia Prevention Guildeline, "China adult dyslipidemia prevention guildeline," Chinese Journal of Cardiology, vol. 35, no. 5, pp. 390-419, 2007.

[4] Z. Lu, W. Kou, B. Du et al., "Effect of Xuezhikang, an extract from red yeast Chinese rice, on coronary events in a Chinese population with previous myocardial infarction," The 
American Journal of Cardiology, vol. 101, no. 12, pp. 16891693, 2008.

[5] X. H. Dai, X. Z. Zhuo, X. Y. Xue, W. M. Yang, X. Q. Yu, and Y. X. Zhou, "Xuezhikang capsule for unstable angina pectoris," Traditional Chinese Drug Research \& Clinical Pharmacology, vol. 10, no. 4, pp. 202-204, 1999.

[6] W. H. Wang, H. Zhang, Y. L. Yu, Z. C. Ge, C. Xue, and P. Y. Zhang, "Effect of Xuezhikang for patients with acute coronary syndrom complicated with different serum lipid levels," Chinese Journal of Integrative Medicine, vol. 24, no. 12, pp. 1073-1076, 2004.

[7] R. G. Ye and Z. Y. Lu, Internal Medicine, People's Medical Publishing House, 6th edition, 2005.

[8] Bureau of Drug Administration of People's Republic of China, "Cardiovascular medicine clinical research guilding principles," 1993.

[9] R. S. Gao and Z. J. Chen, "Further Improving the understanding of unstable angina pectoris," Chinese Journal of Cardiology, vol. 22, no. 4, article 243, 1994.

[10] Chinese Society of Cardiology, "Diagnosis and treatment recommendation for unstable angina pectoris," Chinese Journal of Cardiology, vol. 28, no. 6, pp. 409-412, 2000.

[11] E. Braunwald, E. M. Antman, J. W. Beasley et al., "ACC/AHA guidelines for the management of patients with unstable angina and non-ST-segment elevation myocardial infarction: executive summary and recommendations: a report of the American College of Cardiology/American Heart Association task force on practice guidelines (committee on the management of patients with unstable angina)," Circulation, vol. 102, no. 10, pp. 1193-1209, 2000.

[12] Y. Y. Zheng, Chinese Herbal Medicine Clinical Research Guilding Principles (for trial implementation), Chinese Medicine and Technology Publishing House, 2002.

[13] H. Z. Chen, Practice Internal Medicine, The People's Medical Publishing House, Beijing, China, 10th edition, 2005.

[14] D. S. Goodman, S. B. Hulley, L. T. Clark et al., "Report of the national cholesterol education program expert panel on detection, evaluation, and treatment of high blood cholesterol in adults," Archives of Internal Medicine, vol. 148, no. 1, pp. 3669, 1988.

[15] S. M. Grundy, D. Bilheimer, A. Chait et al., "Summary of the second report of the National Cholesterol Education Program (NCEP) Expert Panel on Detection, Evaluation, and Treatment of High Blood Cholesterol in Adults (Adult Treatment Panel II)," JAMA, vol. 269, no. 23, pp. 3015-3023, 1993.

[16] X. Fang, Z. L. Wang, T. H. Ning et al., "Prevent and treatment recommendation for dyslipidemia," Chinese Journal of Cardiology, vol. 25, no. 3, pp. 169-175, 1997.

[17] J. I. Cleeman, "Executive summary of the third report of the National Cholesterol Education Program (NCEP) expert panel on detection, evaluation, and treatment of high blood cholesterol in adults (adult treatment panel III)," JAMA, vol. 285, no. 19, pp. 2486-2497, 2001.

[18] S. M. Grundy, J. I. Cleeman, C. N. Bairey Merz et al., "Implications of recent clinical trials for the national cholesterol education program adult treatment panel III guidelines," Circulation, vol. 110, no. 2, pp. 227-239, 2004.

[19] S. C. Smith, J. Allen, S. N. Blair et al., "AHA/ACC guidelines for secondary prevention for patients with coronary and other atherosclerotic vascular disease: 2006 Update," Circulation, vol. 113, no. 19, pp. 2363-2372, 2006.

[20] J. P. T. Higgins and S. Green, "Cochrane handbook for systematic reviews of interventions, version 5.0.2 [updated
September 2009]," The Cochrane Collaboration, 2009, http://www.cochrane-handbook.org/.

[21] L. S. Gao and Y. Liao, "Clinical observation of Xuezhikang and fluvastatin sodium capsules on serum lipid," Modern Journal of Integrated Traditional Chinese and Western Medicine, vol. 12, no. 23, article 2528, 2003.

[22] S. Q. Guan, "Long-term effect of Xuezhikang for patients with coronary heart diseae complicated with hyperlipidemia," Guide of China Medicine, no. 1, pp. 62-63, 2010.

[23] S. T. Huang, X. L. Chen, and J. Zhang, "Clinical observation of Xuezhikang for patients with coronary heart disease complicated with hyperlipidemia," Proceeding of Clinical Medicine, no. 3, pp. 193-195, 2005.

[24] S. X. Huang, J. Z. Yin, M. Pan, and Q. L. Liang, "Effect of Xuezhikang for aged patients with angina pectoris complicated with dyslipidemia," Contempoary Medicine, vol. 13, pp. 128-129, 2009.

[25] W. H. Jiang and Z. D. Cai, "Clinical observation of simvastatin for 45 patients with hyperlipidemia," Medical Information, no. 10, pp. 699-700, 2001.

[26] B. Li, S. Y. Hu, X. Wu, H. L. Xu, H. M. Zhang, and L. Wang, "Effect of Xuezhikang for patients with coronary heart disease on anti-oxidation and anti-imflammation," Progress in Modern Biomedicine, no. 12, pp. 2289-2291, 2011.

[27] H. Lin, L. S. Yang, and D. R. Zheng, "Effect of simvastatin and Xuezhikang for patients with unstable angina pectoris," China Medical Herald, vol. 6, no. 23, pp. 58-59, 2009.

[28] D. Q. Lou, B. Liu, W. G. Yan, X. B. Hu, and Y. Wu, "Effect of Xuezhikang for patients with unstable angina pectoris," Clinical Medicine, vol. 28, no. 3, pp. 23-24, 2008.

[29] W. Ma and Y. X. Teng, "Effect of Xuezhikang for patients with coronary heart disease complicated with hyperlipidemia on endothelial function and C response protein," Liaoning Yi Yao, vol. 20, no. 2, pp. 22-24, 2005.

[30] B. L. Qi, G. J. Zhang, and X. X. Suo, "Efficiency rate of Xuezhikang for angina pectoris," Chinese Journal of Primary Medicine and Pharmacy, vol. 8, no. 6, p. 547, 2001.

[31] X. B. Shang, "Clinical observation of Xuezhikang and atorvastatin for patients with coronary heart disease complicated with dyslipidemia on serum lipid and hemorheology," Guangxi Medical Journal, vol. 29, no. 8, pp. 1158-1159, 2007.

[32] J. Wang and M. Y. Xiao, "Long term effect of Xuezhikang and aspirine for 65 patients with coronary heart disease complicated with hyperlipidemia," New Medicine, vol. 31, no. 10, pp. 596-597, 2000.

[33] L. Yan and X. M. Li, "Effect of Xuezhikang for aged patients with coronary heart disease complicated with dyslipidemia on serun lipid modification," Sichuan Medical Journal, vol. 28, no. 11, pp. 1232-1233, 2007.

[34] X. D. Yan, "Clinical observation of Xuezhikang for 88 patients with unstable angina pectoris," Chinese Community Doctors. Comprehensive Edition, vol. 8, no. 15, p. 37, 2006.

[35] H. Yu, Y. L. Cui, S. Y. Wang, and B. Han, "Effect of Xuezhikang for patients with coronary heart diseae complicated with hyperlipidemia on fibrinolytic function," Chinese Journal of Coal Industry Medicine, vol. 5, no. 1, pp. 61-62, 2002.

[36] H. Y. Zhang, "Efficiency of fluvastatin and Xuezhikang for coronary heart disease," China Practical Medicine, no. 22, pp. 115-116, 2010.

[37] X. Zhang, "Clinical effect of atorvastatin calcium tablets for coronary heart disease complicated with hyperlipidemia," Hebei Medical Journal, vol. 33, no. 6, pp. 882-883, 2011. 
[38] C. S. Zhou, W. Z. He, R. Liu, K. Pan, and R. J. Liu, "Effect of Xuezhikang for patienrs with acute coronary syndrome on vascular endothelial function," Chinese Magazine of Clinical Medicinal Professional Research, no. 71, p. 44, 2003.

[39] M. Xu, "Clinical comparative study on treatment of unstable angina pectoris disease with lipid-reducing hongqu Xuezhikang and atorvastatin," Shandong University of traditional Chinese Medicine, 2005.

[40] J. Liu, J. Zhang, Y. Shi, S. Grimsgaard, T. Alraek, and V. Fønnebø, "Chinese red yeast rice (Monascus purpureus) for primary hyperlipidemia: a meta-analysis of randomized controlled trials," Chinese Medicine, vol. 1, article 4, 2006.

[41] C. Gong, S. L. Huang, J. F. Huang et al., "Effects of combined therapy of Xuezhikang Capsule and Valsartan on hypertensive left ventricular hypertrophy and heart rate turbulence," Chinese Journal of Integrative Medicine, vol. 16, no. 2, pp. 114-118, 2010.

[42] L. Lu, J. Z. Zhou, L. Wang, and T. X. Zhang, "Effects of Xuezhikang and Pravastatin on circulating endothelial progenitor cells in patients with essential hypertension," Chinese Journal of Integrative Medicine, vol. 15, no. 4, pp. 266-271, 2009.

[43] X. F. Fan, Y. Q. Deng, L. Ye et al., "Effect of Xuezhikang Capsule on serum tumor necrosis factor- $\alpha$ and interleukin- 6 in patients with nonalcoholic fatty liver disease and hyperlipidemia," Chinese Journal of Integrative Medicine, vol. 16, no. 2, pp. 119-123, 2010.

[44] M. A. Pfeffer, F. M. Sacks, L. A. Moyé et al., "Cholesterol and recurrent events: a secondary prevention trial for normolipidemic patients," The American Journal of Cardiology, vol. 76, no. 9, pp. 98C-106C, 1995.

[45] Z. L. Lu, "Advance in basic and clinical research of Xuezhikang Capsule: a commentary on the Chinese coronary secondary prevention study," Chinese Journal of Integrative Medicine, vol. 12, no. 2, pp. 85-87, 2006. 


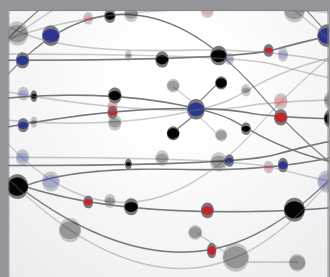

The Scientific World Journal
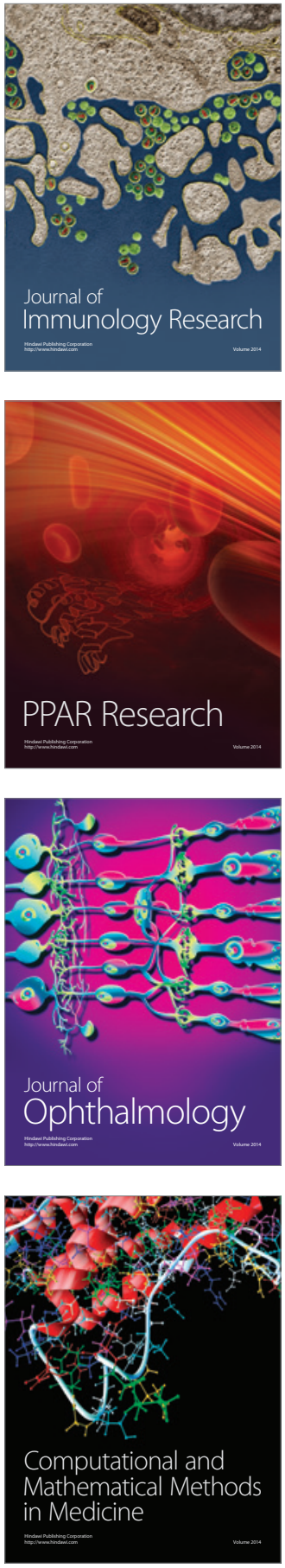

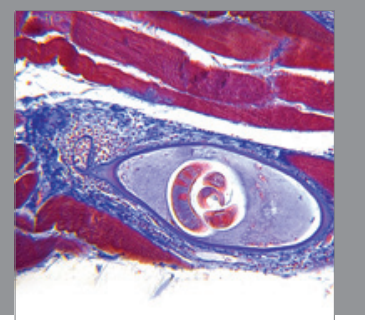

Gastroenterology

Research and Practice
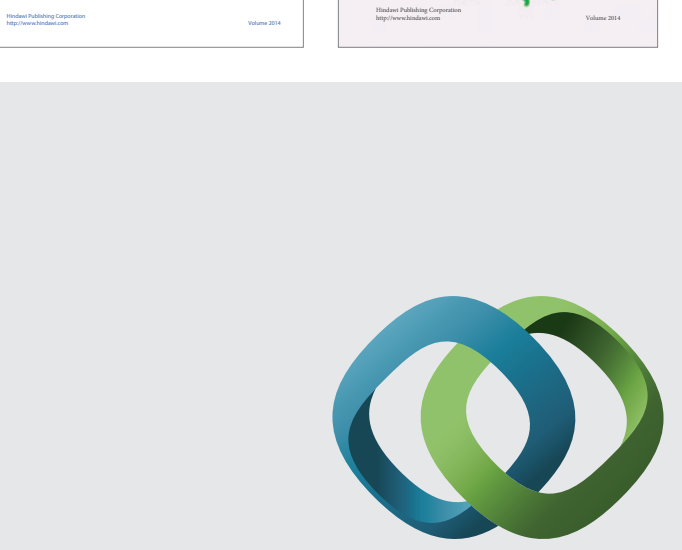

\section{Hindawi}

Submit your manuscripts at

http://www.hindawi.com
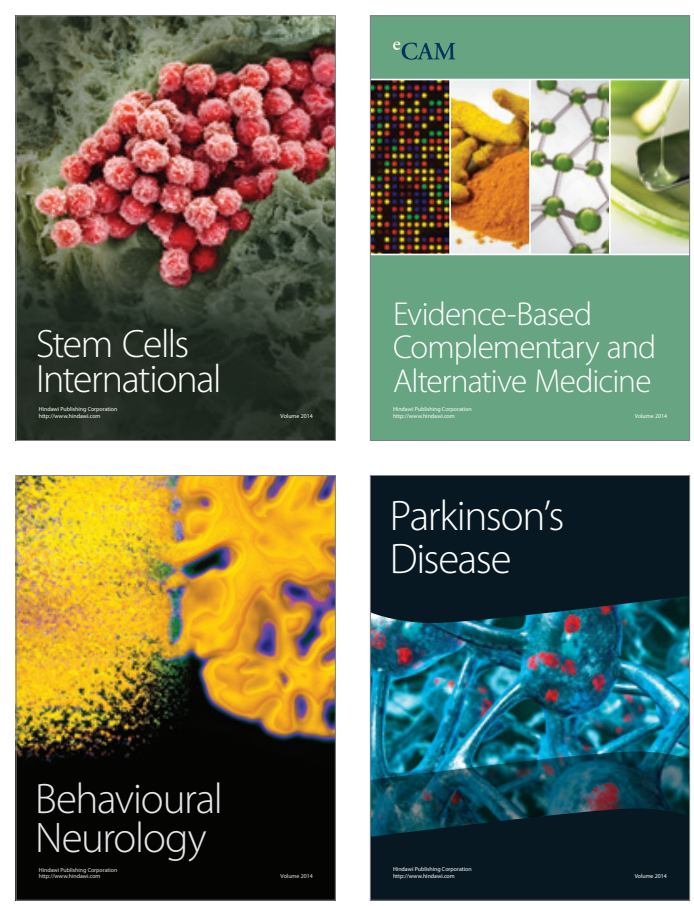

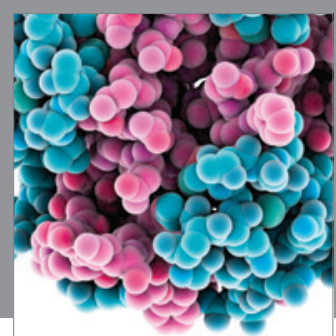

Journal of
Diabetes Research

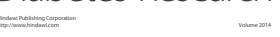

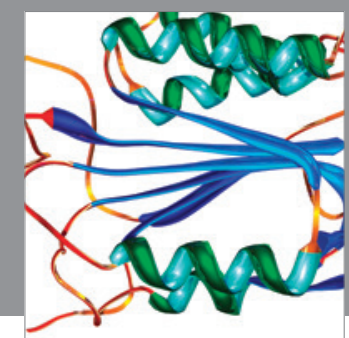

Disease Markers
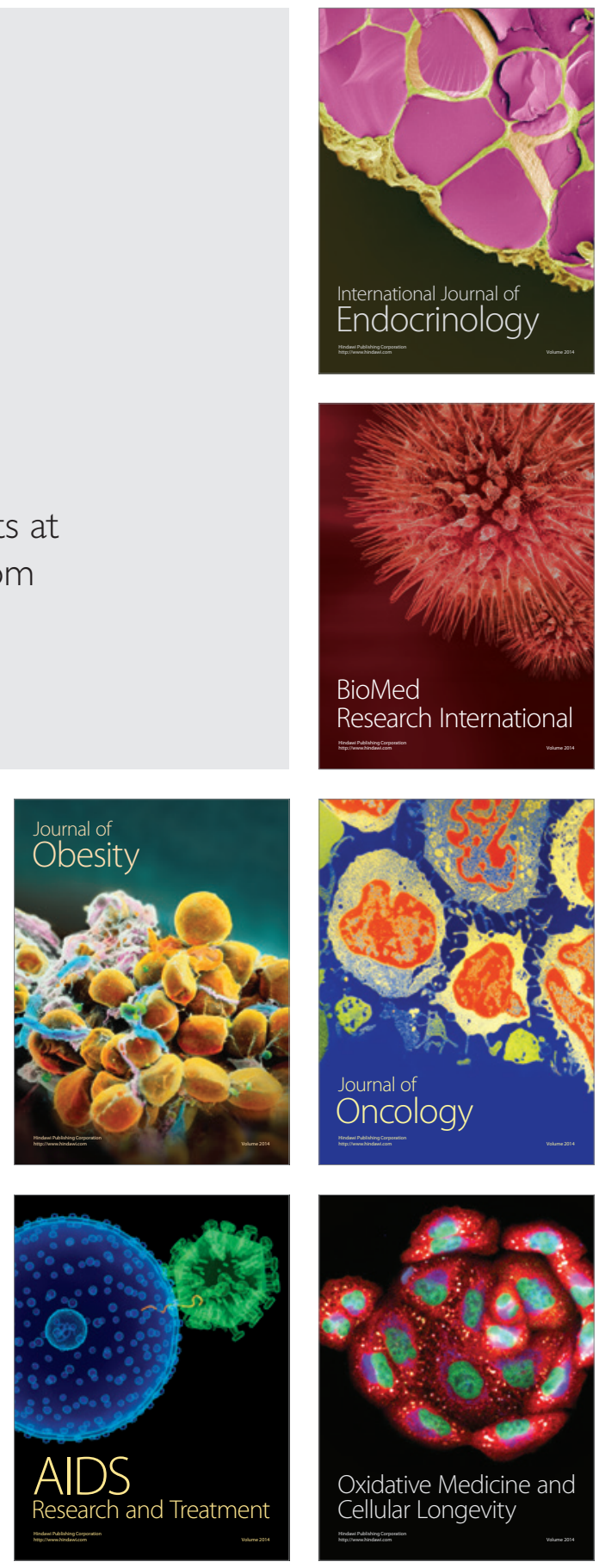